\title{
ESTUDIO SOCIOFONÉTICO DE LOS TONEMAS DE LAS INTERROGATIVAS ABSOLUTAS EN EL CASTELLANO DE GALICIA
}

\author{
Susana PÉrez Castillejo \\ Universidad de Minnesota, Twin Cities \\ perez050@umn.edu
}

\begin{abstract}
Resumen
A partir de un corpus de interrogativas absolutas producidas por 24 hablantes, este trabajo analiza los efectos suprasegmentales del contacto entre castellano y gallego. Adoptando una metodología sociofonética y bajo el marco teórico del sistema métrico autosegmental, se examinan los tonemas interrogativos en función del contexto pragmático, formalidad de la tarea y la exposición al gallego de cada participante. Los resultados revelan variación estilística, indicativa de la estigmatización de rasgos vernáculos, y un cambio de relaciones prosódico-pragmáticas inducido por el contacto con el gallego.
\end{abstract}

PALABRAS CLAVE: castellano de Galicia, contacto gallego-español, entonación, interrogativas absolutas, relaciones prosódicopragmáticas.

\section{Introducción}

Para ser tan reconocible perceptualmente, sorprende la escasez de descripciones acústicas de la entonación del castellano ${ }^{1}$ hablado en Galicia. Por un lado están las listas dialectológicas (García, 1976; García y Blanco, 1998; Porto Dapena, 2001) que incluyen la entonación entre los rasgos peculiares de esta región. Pese a constituir un excelente

\footnotetext{
${ }^{1}$ En este trabajo se utilizan los términos español y castellano indistintamente. Las referencias a una variedad particular de esta lengua se matizan de alguna forma, como, por ejemplo, el castellano de Galicia o el castellano del centro de la Península Ibérica.
}

KEY WORDS: Galician Spanish, language contact in Galicia, intonation, absolute interrogatives, prosodic-pragmatic relationships.
From a corpus of absolute interrogatives produced by 24 speakers, the present study analyzes the suprasegmental effects of the applies a socio-phonetic methodology and the Autosegmental-Metrical theoretical framework to examine interrogative tonemes acand the participants' exposure to Galician. Results reveal stylistic variation, indicative of the stigmatization of vernacular features, and a contact-induced change in prosodicpragmatic relationships. 
punto de partida para investigar los contornos entonativos del español de esta variedad, dichas descripciones son por necesidad escuetas y solo se basan en las impresiones auditivas de los autores. Por otro lado se encuentra el estudio de Castro (2003), el único trabajo experimental hasta el momento sobre la entonación del castellano de Galicia. Gracias a dicho estudio sabemos que el origen de algunas de las diferencias entonacionales de esta variedad pueden explicarse por el contacto histórico entre gallego y castellano. Sin embargo, la autora solo muestra conclusiones preliminares sin un análisis cuantitativo de los efectos de dicho contacto. Además, el análisis de Castro (2003) no se adscribe al marco teórico del sistema métrico autosegmental ${ }^{2}$ que adoptan la mayoría de las investigaciones más recientes sobre entonación del español, lo cual en cierto modo limita la comparación interdialectal con variedades monolingües.

Los efectos suprasegmentales del contacto entre el castellano y otras lenguas del continuo ibero-romance noroccidental de la Península Ibérica se han explorado también en Asturias y Cantabria, dos comunidades autónomas cercanas a Galicia (López Bobo et alii, 2008, en Asturias, y López Bobo y Cuevas Alonso, 2009 y 2010, en Cantabria). A raíz de estos estudios sabemos que una consecuencia de este tipo de contacto es el uso de un tonema ${ }^{3}$ descendente $\mathrm{H}+\mathrm{L}^{\star} \mathrm{L} \%$ y otro ascendente-descendente - también llamado circunflejo $-\mathrm{H}^{\star} \mathrm{HL} \%$ en interrogativas absolutas neutras ${ }^{4}$, frente al tonema ascendente $L^{\star} \mathrm{H} \%$ más extendido en otras variedades del español europeo para este tipo de oración (Face, 2008; Estebas y Prieto, 2010). Las representaciones esquemáticas de la Figura 1 ilustran el movimiento de la curva de entonación en los tres tonemas mencionados. El espacio sombreado representa la última sílaba tónica del enunciado y el espacio en blanco corresponde al material pre- y postónico.

\footnotetext{
${ }^{2}$ El sistema métrico autosegmental, desarrollado a partir del trabajo de Pierrehumbert (1980) asume que la curva melódica puede analizarse fonológicamente en una serie de unidades, entre ellas los acentos tonales y los tonos de frontera. Los acentos tonales se asocian con las sílabas tónicas del enunciado (asociación que se marca con un asterisco ${ }^{\star}$ ) y se especifican como L, si el tono se mantiene bajo, o $\mathrm{H}$, si se mantiene alto. Las combinaciones bitonales $\mathrm{L}+\mathrm{H}$ y $\mathrm{H}+\mathrm{L}$ representan el movimiento de la curva ascendente y descendente respectivamente. En cuanto a los tonos de frontera (los que ocurren justo al final de la frase), generalmente se asume una representación binaria: L\% especifica una caída final de la curva melódica y $\mathrm{H} \%$ representa un final ascendente.

${ }^{3}$ Se entiende por tonema la configuración de la curva melódica desde el inicio de la última sílaba tónica hasta el final del enunciado.

${ }^{4}$ Las interrogativas absolutas (también llamadas declarativas o totales) son aquellas en las que se espera una respuesta de tipo sí/no. Se denominan neutras cuando su función pragmática es la petición de información, frente a las no neutras con funciones comunicativas como la confirmación de información o la invitación, entre otras (Escandell Vidal, 1999).
} 


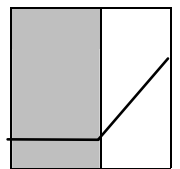

$\mathrm{L}^{\star} \mathrm{H} \%$

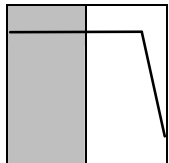

$\mathrm{H}^{\star} \mathrm{HL} \%$

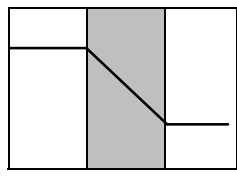

$\mathrm{H}+\mathrm{L}^{\star} \mathrm{L} \%$

Figura 1. Tonemas ascendente, circunflejo y descendente según el sistema de transcripción Sp_ToBI (Aguilar, De la Mota y Prieto, 2009)

Si el contacto entre lenguas romances en el noroeste de España ha producido variación intradialectal en la entonación interrogativa del castellano de Asturias y Cantabria, es plausible que lo mismo ocurra en el castellano de Galicia. Sin embargo, hasta el momento no se ha analizado un corpus suficientemente grande y con la metodología adecuada para esclarecer si tal variación se da en efecto en Galicia y, de ser así, cuál es su alcance geográfico y social.

Los tonemas descendente y circunflejo se han documentado en las interrogativas absolutas en gallego. Fernández Rei et alii (2005) reportan un tonema interrogativo en Santiago de Compostela que podría analizarse como $\mathrm{H}+\mathrm{L}^{*} \mathrm{~L} \%$ según las convenciones del sistema métrico autosegmental desarrolladas para el español, el Sp_ToBI propuesto por Estebas y Prieto (2009). Fernández Rei (2007) propone un tonema ${ }_{i} \mathrm{H}+\mathrm{L}^{*} \mathrm{~L}-\mathrm{L} \%$ para las interrogativas en gallego de Carballo (localidad cercana a La Coruña), un tonema $\mathrm{H}+\mathrm{L}^{*} \mathrm{~L}-\mathrm{L} \%$ en Camelle (en la costa oeste a $100 \mathrm{~km}$ de La Coruña) y una configuración final $\mathrm{L}+\mathrm{H}^{\star} \mathrm{H}-\mathrm{L} \%$ en la comarca del Bajo Miño (en la provincia de Pontevedra, al suroeste de Galicia). Contornos similares, aunque con un análisis fonológico ligeramente diferente, $\mathrm{H}+\mathrm{L}^{*} \mathrm{~L} \%$ y $\mathrm{L}+\mathrm{H}^{*} \mathrm{HL} \%$, se han reportado en el castellano del centro de España (Estebas y Prieto, 2010), variedad que se toma como estándar del español entre los castellanoparlantes de Galicia (Beswick, 2007; Regueira Fernández, 2004). Sin embargo, el contexto pragmático en el que aparecen estos dos patrones en Madrid es diferente del que muestran en el gallego de las localidades mencionadas: en castellano, $\mathrm{H}+\mathrm{L}^{\star} \mathrm{L} \%$ ocurre en interrogativas absolutas imperativas (ej., ¿queréis callar?) o que confirman una información conocida, mientras que $\mathrm{L}+\mathrm{H}^{\star} \mathrm{HL} \%$ se encuentra en vocativos y peticiones insistentes (ej., ¿verdad que vendrás?). Ninguna de estas dos configuraciones finales aparece en las interrogativas absolutas neutras del castellano central, oraciones que se caracterizan por un tonema ascendente analizado como $\mathrm{L}^{\star} \mathrm{HH} \%$ por Estebas y Prieto (2010). La variación de tonemas interrogativos en el castellano central viene determinada pues por la función pragmática de la pregunta.

Si efectivamente la entonación del castellano de Galicia presenta influjo del gallego, como han propuesto García (1976), Porto Dapena (2001) y Castro (2003), una posible 
consecuencia sería que los tonemas encontrados en las interrogativas absolutas neutras en gallego también afloraran en la misma modalidad oracional en el castellano de Galicia. La presente investigación examina un corpus de interrogativas absolutas producidas por 24 gallegos cuya lengua habitual es el castellano en contextos pragmáticos neutros (de petición de información) y no neutros (de confirmación de información y de pregunta imperativa). El objetivo es determinar si, como resultado del contacto histórico entre gallego y castellano en Galicia, las interrogativas absolutas neutras producidas por los castellanoparlantes de esta región presentan configuraciones tonémicas propias de estas oraciones en gallego. Este tipo de interferencia entre lenguas es consistente con el modelo que postula Silva Corvalán (1994), según el cual la transferencia de rasgos lingüísticos entre sistemas en contacto solo puede darse si existe entre ambos un paralelismo a nivel de superficie en torno a dichos rasgos. Una forma de transferencia posible es la ampliación del uso pragmático de un patrón existente en ambas lenguas, pero con usos más limitados en una de ellas. En el caso que nos ocupa, el tonema descendente $\mathrm{H}+\mathrm{L}^{\star} \mathrm{L} \%$ con función interrogativa existe tanto en castellano como en gallego, pero los contextos pragmáticos en los que puede aparecer son más restringidos en la primera que en la segunda lengua. Silva Corvalán además matiza que mientras que la estructura lingüística de ambas lenguas determina qué elementos se trasfieren por contacto, la historia sociolingüística de los hablantes incide en el grado de difusión de la transferencia. Aplicando las predicciones de este modelo al caso del castellano de Galicia, cabe esperar heterogeneidad socialmente motivada en el grado de incidencia del gallego en las configuraciones tonémicas de las interrogativas absolutas neutras del castellano hablado en la región, si es que en efecto hay influencia.

Resumiendo, pues, en base al modelo de transferencia por contacto de lenguas de Silva Corvalán (1994), este trabajo testea la hipótesis de que los tonemas que caracterizan la entonación interrogativa absoluta neutra en gallego se han extendido al castellano hablado en Galicia, pero el grado en que dicha extensión ocurre puede estar sujeto a variación sociolingüística. Las preguntas de investigación que guían este estudio son:

1. ¿Qué tonemas se encuentran en las interrogativas absolutas del castellano de Galicia?

2. La distribución de los tonemas encontrados ¿se rige por el contexto pragmático o por factores relacionados con el contacto histórico entre castellano y gallego en esta región? 


\section{Antecedentes históricos y estudios previos}

El origen del contacto histórico entre el castellano y las lenguas ibero-romances del noroeste de la Península Ibérica suele situarse en el siglo XIII, a raíz de la consolidación del liderazgo político y económico del Reino de Castilla (Ramallo, 2007: 22). En el caso del territorio que hoy conocemos como Galicia, la expansión del área de influencia lingüística castellana fue un proceso gradual, impulsado en sus comienzos por las élites sociales. A lo largo de varios siglos, el castellano se fue erigiendo como lengua de la administración y de cultura, mientras que el uso del gallego se fue restringiendo a los ámbitos privados. Pese a los intentos de revitalización del gallego a comienzos del siglo XX, la política lingüística franquista (1939-1975) - que favorecía el castellano como vehículo de promoción social (Bouzada Fernández, 2003: 325) - produjo una seria interrupción en la transmisión intergeneracional del gallego. Con la llegada de la democracia (1978), el gallego recobró su presencia en la esfera pública: el gallego es cooficial con el español en Galicia y el Estatuto de Autonomía de Galicia (1981) contiene una serie de políticas sociales y educativas que promueven el bilingüismo entre la sociedad gallega. A pesar de la presencia constante del castellano en Galicia desde la Edad Media, el gallego siguió siendo la lengua de uso habitual para la mayoría hasta el siglo XX (García, 1976), favoreciendo la transferencia interlingüística y dando lugar a una variedad del castellano fácilmente reconocible por hablantes de otras regiones de España. Las investigaciones del castellano hablado en Galicia, sin embargo, son relativamente escasas y aún más en lo concerniente a la entonación.

Como mencionamos antes, las descripciones de la entonación del castellano hablado en Galicia disponibles hasta ahora se limitan a tres breves referencias en listas de rasgos dialectales de esta región (García, 1976; García y Blanco, 1998; Porto Dapena, 2001) y un único estudio experimental (Castro, 2003). Las descripciones de García (1976), García y Blanco (1998) y Porto Dapena (2001) incluyen la entonación entre los elementos perceptualmente más distintivos del castellano de Galicia y concuerdan en la relevancia que asignan a la interferencia del gallego en el desarrollo de esta particularidad. Sin embargo, difieren en el grado de difusión que otorgan a dicha interferencia: para los dos primeros la entonación del español de Galicia "es exactamente la entonación del gallego" (García, 1976: 331), mientras que Porto Dapena (2001) matiza que existe variación geográfica y diastrática en el grado en que los castellanohablantes de la región muestran una pronunciación más o menos agallegada. Ninguna de estas dos descripciones especifica en qué consiste una entonación agallegada ni tampoco hacen referencia concreta a la entonación interrogativa, que es el objeto del presente estudio. 
Castro (2003) analiza el contorno entonacional de una serie de oraciones extraídas de un corpus de habla espontánea en gallego y en castellano, emitidas por hablantes de gallego, hablantes de castellano de Galicia y hablantes de castellano de regiones monolingües. A partir de sus observaciones la autora concluye que la entonación del castellano de Galicia presenta ciertos rasgos atribuibles al contacto con el gallego, entre ellos: una mayor intensidad asociada con las sílabas tónicas, un descenso menos pronunciado al final de los enunciados declarativos y el mantenimiento de las frecuencias altas hasta el acento nuclear en las interrogativas. El valor del trabajo de Castro (2003) reside en señalar rasgos específicos que distinguen los contornos entonativos de esta variedad del español con respecto a otras. La autora arguye el contacto histórico con el gallego como explicación de estas diferencias, pero el diseño de su estudio no le permite explorar de manera adecuada los factores sociales que pueden corroborar dicha propuesta. Dos limitaciones de su metodología son que carece de un análisis cuantitativo de los rasgos observados y que, salvo la variedad de habla, no hay ningún otro dato sobre la historia sociolingüística de los informantes. El trabajo de Castro no puede responder, por tanto, la cuestión de si los rasgos que comparten los contornos en gallego y en español de Galicia en su muestra se pueden generalizar a la población castellanoparlante de esta región o si existe variación en el grado en que estos hablantes presentan una entonación más o menos agallegada.

Hay dos estudios de la entonación del español del noroeste de la Península Ibérica que encuentran variación de contornos entonativos en función de la mayor o menor castellanización de la zona analizada. López Bobo et alii (2008) comparan oraciones declarativas e interrogativas de dos zonas de Asturias: el centro - más poblado y con mayor influjo de castellanohablantes - y el occidente - en el que persisten rasgos prosódicos típicos del continuo lingüístico asturiano. Las diferencias entre ambas modalidades de habla se observan sobre todo en las interrogativas. Mientras que en ambas zonas se observan tonemas descendentes, las interrogativas del occidente mantienen mayor altura tonal en el material pretonemático y presentan desacentuación con mayor frecuencia que las interrogativas de la zona central.

López Bobo y Cuevas Alonso (2009) realizan el mismo tipo de comparación en Cantabria, región al este de Asturias. Al examinar enunciados declarativos e interrogativos de una zona rural y de otra urbana, encuentran que la primera mantiene patrones entonativos que los autores denominan norteños, mientras que el habla urbana exhibe patrones castellanos similares a los de otros dialectos del español europeo. Las diferencias que observan los autores entre ambas modalidades de habla son la presencia de un tonema descendente en las interrogativas del patrón norteño frente al tonema ascendente

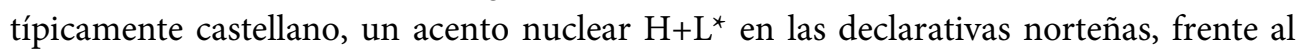


acento nuclear $\mathrm{L}^{*}$ típico de las declarativas norteñas, y la falta de consistencia en la asociación de un pico tonal con cada sílaba tónica.

Un dato al que no hacen referencia los estudios de López Bobo et alii (2008) y de López Bobo y Cuevas Alonso (2009) es el contexto pragmático en el que ocurrieron las interrogativas analizadas en su muestra. Sabemos que el tonema descendente que estos autores encuentran en las interrogativas de las variedades menos castellanizadas también se da en el castellano más extendido por la Península Ibérica, aunque con diferente función pragmática (Face, 2008). Estebas y Prieto (2010) constatan en un corpus de habla semi-espontánea de Madrid que mientras que el tonema L*HH\% caracteriza las preguntas absolutas de tipo informativo, el tonema descendente $\mathrm{H}+\mathrm{L}^{\star} \mathrm{L} \%$ señala las preguntas con una función imperativa o que confirman información conocida. La falta de control del contexto pragmático de los enunciados interrogativos en los estudios de Asturias y Cantabria debilita en cierto modo la explicación de los tonemas descendentes de las interrogativas como producto del contacto con las variedades ibero-romances del noroeste de España, ya que no se puede determinar si los hablantes produjeron tonemas descendentes por influencia de otra lengua romance o porque interpretaron la función de la pregunta como imperativa o de confirmación.

Esta limitación se supera en un estudio posterior también en Cantabria (López Bobo y Cuevas Alonso, 2010), en el que se controla la interpretación pragmática de los estímulos presentados a los hablantes. Los autores observan que, junto al tonema ascendente propio del castellano del centro peninsular, las preguntas informativas también presentan un tono de frontera circunflejo HL\% observado en la variedad romance autóctona de la zona. Este tono de frontera se atestigua en el castellano de Madrid, aunque en contextos pragmáticos diferentes (Estebas y Prieto, 2010). La intención del estudio de López Bobo y Cuevas Alonso (2010) es tan solo describir la variación entonacional observada y no explicar su origen. Sin embargo, podríamos decir que sus resultados son consistentes con el modelo de Silva Corvalán (1994), según el cual una forma lingüística amplía su repertorio de funciones pragmáticas por contacto con otra lengua en la que dicha forma tiene un uso más generalizado.

La presente investigación replica la metodología empleada por Estebas y Prieto (2010) y López Bobo y Cuevas Alonso (2010) para describir las configuraciones tonémicas de las interrogativas absolutas del castellano de Galicia, pero va más allá al ofrecer una explicación de la variación a partir de factores sociolingüísticos. Al contar con un corpus más amplio y con más detalles de la historia sociolingüística de los informantes, este estudio se encuentra en una buena posición para determinar el alcance de la posible variación prosódico-pragmática provocada por el contacto histórico entre gallego y castellano. 


\section{Metodología}

\subsection{Participantes}

Las estadísticas sobre usos lingüísticos en Galicia indican que la población castellanoparlante en Galicia tiende a concentrarse en las áreas urbanas, pertenecer a las clases acomodadas y tener un alto nivel educativo (O'Rourke, 2006: 183). Estos fueron los criterios para seleccionar la muestra de la presente investigación, que se compone de 24 informantes (14 hombres y 10 mujeres) originarios de tres ciudades gallegas, La Coruña, Santiago y Vigo, con edades comprendidas entre los 18 y 64 años. Todos los participantes habían completado - o estaban cursando en el momento de la investigación estudios superiores, pero ninguno tenía formación lingüística que pudiera alertarle sobre los objetivos de la investigación. A través de un cuestionario sociolingüístico se constató que el castellano era la lengua dominante de los participantes, aunque todos habían estado expuestos a la lengua gallega en mayor o menor grado.

\subsection{Obtención de los datos}

\subsubsection{Cuestionario sociolingüístico}

Los participantes respondieron sobre una escala Likert a una serie de preguntas acerca de su experiencia con y habilidad en la lengua gallega. La encuesta, que se distribuyó por internet o en papel, se diseñó para extraer información sobre la historia sociolingüística de cada participante (lugar donde nació, dónde creció, edad a la que empezó a aprender gallego, dónde lo aprendió, etc.). Dada la variación observada en los contornos entonacionales del gallego de distintas localidades (Fernández Rei, 2007), el cuestionario sociolingüístico también pedía información sobre el origen de los padres y abuelos de cada uno de los participantes, el lugar de residencia y la asiduidad con que los abuelos interactuaban con cada participante durante la infancia, así como los lugares en que cada participante había residido a lo largo de su vida y la duración de la permanencia en dicho lugar. En la medida de lo posible, el cuestionario trató de distinguir entre exposición al gallego en un ambiente académico y en un entorno social o familiar, con el fin de capturar la complejidad del bilingüismo en esta región en la que es posible alcanzar una alta habilidad en la lengua gallega sin haber tenido instrucción formal en dicha lengua. Pese a la clara limitación de ser datos auto-reportados, consideramos que esta metodología es válida para indicar el grado de exposición al gallego de cada individuo. Los datos de este cuestionario se cotejaron con los obtenidos a través de otras dos tareas: una tarea formal de lectura de frases y otra de situaciones hipotéticas destinada a obtener muestras de habla semi-espontánea. 


\subsubsection{Lectura de frases}

Siguiendo el modelo de Henricksen (2010), se diseñaron 24 combinaciones de dos palabras léxicas que se insertaron en 48 contextos distintos para provocar su lectura como declarativas neutras y como interrogativas absolutas neutras (peticiones de información) (véase Apéndice A). Es decir, cada participante leyó 24 pares de oraciones segmental y sintácticamente idénticas, pero diferentes en el patrón entonativo requerido por el contexto en el que cada elemento del par se insertaba. Los 48 enunciados se presentaron en orden aleatorio a través de power point con una diapositiva para cada frase. Al principio de la presentación se insertaron tres oraciones de práctica que no forman parte del estudio. Las instrucciones para los participantes fueron que leyeran el contenido de cada diapositiva en voz alta dos veces de la forma más natural posible y que, si sentían que se habían equivocado, volvieran a repetir la diapositiva en cuestión. Los participantes controlaron la velocidad de la presentación y no interactuaron con ningún otro individuo mientras completaban esta prueba. Para el presente trabajo solo se analizan las frases interrogativas, con un total de 1152 casos (24 oraciones x 2 repeticiones $\mathrm{x} 24$ hablantes).

Además del contexto de interpretación pragmática, en el diseño de los enunciados se controló la distancia en sílabas entre las dos sílabas tónicas de la oración $(0,1$ ó 2 sílabas) y distancia en sílabas entre la última sílaba tónica y el final de la oración (0, 1 ó 2 sílabas). El objetivo principal de la tarea de lectura fue el de obtener una muestra suficiente de enunciados con estructura silábica controlada para poder analizar las características fonéticas de los contornos entonacionales de las declarativas e interrogativas neutras en el castellano de Galicia. El número y la naturaleza contralada de los casos obtenidos en este tipo de tarea no es posible obtener en un corpus de habla espontánea.

\subsubsection{Encuesta de situaciones}

Para obtener ejemplos de enunciados declarativos e interrogativos en un contexto menos formal que la lectura de frases, pero todavía controlando la interpretación pragmática, se replicó la metodología de Estebas y Prieto (2010) y López Bobo y Cuevas Alonso (2010). Los estímulos y las instrucciones sobre cómo aplicar esta metodología se encuentran en línea en el Atlas interactivo de la entonación del español (Prieto y Roseano, 2010). Se trata de una serie de situaciones cotidianas pensadas para producir un tipo de enunciado en particular. El entrevistador lee las situaciones hipotéticas para el informante y le pide que exprese de forma natural lo que diría en tales situaciones. Los 
informantes no tienen acceso al texto escrito, por lo que es posible obtener datos más espontáneos de los que se obtienen en tareas de lecturas.

Los estímulos del modelo completo de la encuesta (70 situaciones) se adaptaron con la ayuda de un colaborador nativo de Galicia (La Coruña) para eliminar los giros o referencias que pudieran considerarse extraños a este dialecto del castellano. Para el presente análisis solo se examinan 16 de las 70 situaciones de la encuesta, correspondientes a tres contextos pragmáticos: de petición de información, de pregunta confirmatoria o de pregunta imperativa (véase Apéndice B). Además de obtener datos menos formales, otro objetivo de esta tarea era el de comprobar si el contraste pragmático entre el tonema ascendente y el descendente observado en las interrogativas del castellano del centro de la Península Ibérica ${ }^{5}$ tiene validez o no en el castellano de Galicia. La encuesta de situaciones se administró después de la lectura de frases para todos los participantes.

Las instrucciones de administración de la encuesta se siguieron tal como se describen en el Atlas (Prieto y Roseano, 2010), salvo que por motivos obvios no se administró solo a mujeres de mediana edad, sino a todos los individuos que participaron en el estudio. En cuanto a la recomendación del Atlas de pedir al informante que reformule la respuesta si el investigador la juzga inadecuada para el contexto deseado, esta petición se evitó en la medida de lo posible en la presente investigación y en ningún caso se hizo más de una vez para un determinado contexto. La motivación para esta ligera desviación de las instrucciones del Atlas es la opinión de que la excesiva reformulación puede ser contraproducente para el objetivo principal de la tarea: la obtención de datos semiespontáneos. Como consecuencia de esta decisión, hubo contextos que no produjeron el enunciado deseado (p.ej., se produjo un verbo imperativo cuando se pretendía obtener una interrogativa con función imperativa) y que, por tanto, se eliminaron del análisis. El tamaño relativamente amplio de la muestra contrarresta esta limitación.

La investigación se llevó a cabo en dos localidades gallegas, La Coruña y Santiago de Compostela. La elección de estas localidades fue por conveniencia y porque tradicionalmente las ciudades, frente a las localidades más pequeñas, en Galicia se consideran las zonas con mayor presencia de castellanoparlantes. Las grabaciones se realizaron en una habitación tranquila en la casa o en el lugar de trabajo de cada participante, con un micrófono unidireccional Shure SM10A y una grabadora digital Marantz PMD 660 en formato .wav a $44100 \mathrm{~Hz}$ y 16 bits. Los segmentos correspondientes a las frases interro-

\footnotetext{
${ }^{5}$ Recordemos que en Madrid el tonema ascendente es característico de las preguntas cuya función es la petición de información, mientras que el descendente, de aparecer, se limita a los contextos no neutros de pregunta confirmatoria o imperativa.
} 
gativas de las dos tareas se extrajeron por medio del programa Adobe Audition para su posterior análisis acústico con el programa Praat.

\subsection{Codificación y análisis}

El objeto de análisis es el tonema de cada oración interrogativa, puesto que es en este elemento en el que se han reportado patrones diferentes para el gallego y para el castellano del centro peninsular. Cada oración del corpus se examinó visual y auditivamente, y el contorno de cada tonema se transcribió de acuerdo con las convenciones de Estebas y Prieto (2009), salvo en el análisis del tono de frontera ascendente que se considera en este trabajo como $\mathrm{H} \%$ en lugar de $\mathrm{HH} \%$ (véase $\$ 4.2$. para una representación esquemática de los contornos y una explicación de la discrepancia en torno a $\mathrm{H} \%$ y $\mathrm{HH} \%)$. Las configuraciones tonémicas que se registran en la muestra, tanto en la tarea de lectura como en la encuesta de situaciones, son: $\mathrm{L}^{\star} \mathrm{H} \%, \mathrm{~L}+\mathrm{H}^{\star} \mathrm{H} \%, \mathrm{H}+\mathrm{L}^{\star} \mathrm{L} \%$ y $\mathrm{L}+\mathrm{H}^{\star} \mathrm{HL} \%$.

Cada tonema obtenido de la tarea de lectura se codificó además en función de las siguientes variables lingüísticas y extralingüísticas: distancia en sílabas entre la sílaba nuclear y la prenuclear $(0,1$ ó 2$)$, distancia en sílabas entre la sílaba nuclear y el final de la oración $\left(0,1\right.$ ó 2), primera o segunda repetición de la frase ${ }^{6}$, género del hablante, edad, localidad en la que creció, el ámbito en el que el hablante reporta exposición al gallego (en casa de pequeño, en la escuela o en el trabajo/universidad de adulto) y el grado de exposición al gallego en casa al crecer (si los dos padres hablaban algo de gallego, si solo uno de los padres utilizaba algo de gallego o si ninguno de los padres utilizaba el gallego).

Los datos sobre la estructura silábica de las oraciones no se utilizan en el presente trabajo, ya que se reservan para la descripción fonética de los contornos que se realiza en otro lugar. Con el fin de testear la hipótesis del contacto con el gallego en la que se centra este estudio, las variables sociolingüísticas se sometieron a un análisis de regresión logística binomial con el programa GoldVarb.

\footnotetext{
${ }^{6}$ Dada la hipótesis que testea este trabajo, se espera que la elección de configuración tonémica esté socialmente condicionada. Si hay variación social, puede que también haya variación en función de la formalidad. Asumiendo que la segunda repetición de una forma lingüística es más cuidada o formal que la primera (Labov, 1972), codificamos la variable de la repetición para explorar si tiene algún efecto en esta muestra.
} 


\section{Resultados}

\subsection{Datos obtenidos del cuestionario sociolingüístico}

La Tabla 1 presenta los datos relevantes sobre la historia sociolingüística de los participantes. Para facilitar la exposición, se especifica dónde creció cada participante, pero no se han incluido los nombres específicos de las localidades donde crecieron los padres y abuelos, sino tan solo la provincia (en caso de ser originarios de Galicia) y, si se trata de un área rural, se añade el símbolo $(\mathrm{R})^{7}$. La distinción entre origen rural o urbano viene motivada por el hecho de que, tradicionalmente, se considera que en Galicia las ciudades son predominantemente castellanoparlantes, mientras que las zonas rurales cuentan con mayor población gallegoparlante o que hablan un castellano fuertemente agallegado al haberlo aprendido como segunda lengua (García, 1976). Dada la diversidad lingüística de Galicia, al especificar la provincia y distinguir entre área rural y urbana, el lector puede obtener una mejor idea del tipo de input al que cada participante estuvo expuesto en el seno familiar. Por el mismo motivo, también se reporta si uno o los dos padres del informante no eran gallegos, aunque de nuevo por razones de espacio la información se abrevia con el adverbio fuera ${ }^{8}$. En la columna Exposición al gallego de niño se marca con $X$ si el informante oyó hablar gallego en casa (ya sea de los padres o de los abuelos) o entre sus mejores amigos de la infancia.

De los 24 individuos de este grupo, 10 reportaron venir de familias de origen urbano; de estos 10, la mitad reportó haber escuchado algo de gallego en casa - aunque poco - de pequeño o entre los amigos, dato que sugiere que pese a ser predominantemente castellanoparlantes, en las ciudades gallegas el contacto entre gallego y castellano es todavía una realidad. Dos hechos de la historia sociolingüística de Galicia reflejados en esta muestra son la migración del campo a las zonas más urbanizadas en las últimas décadas y el cambio de política lingüística que se produjo con la aprobación del Estatuto de Autonomía en 1981 y que dio lugar a la generalización de la enseñanza del gallego en Galicia. En cuanto al proceso migratorio, vemos que 14 informantes tenían abuelos originarios de municipios muy pequeños, pero solo 8 tenían padres nacidos en una zona que podría considerarse rural y, por supuesto, todos los individuos de la muestra nacieron y crecieron en un ambiente urbano. En lo que concierne a la política lingüística, en la Tabla 1 se observa que los informantes más jóvenes aprendieron gallego en la

\footnotetext{
${ }^{7}$ Según criterio de la Red Española de Desarrollo Rural (www.redr.es) son municipios rurales los que tienen menos de 150 habitantes $/ \mathrm{km}^{2}$.

${ }^{8}$ Cabe precisar que en todos los casos fuera hace referencia a regiones monolingües de España.
} 
escuela, lo cual implica que, aunque no hayan estado expuestos al gallego en un ambiente familiar, sí que lo han estado en un ambiente académico. Nos referiremos de nuevo a estos datos sobre la historia sociolingüística de los participantes más adelante cuando interpretemos los resultados de las pruebas lingüísticas.

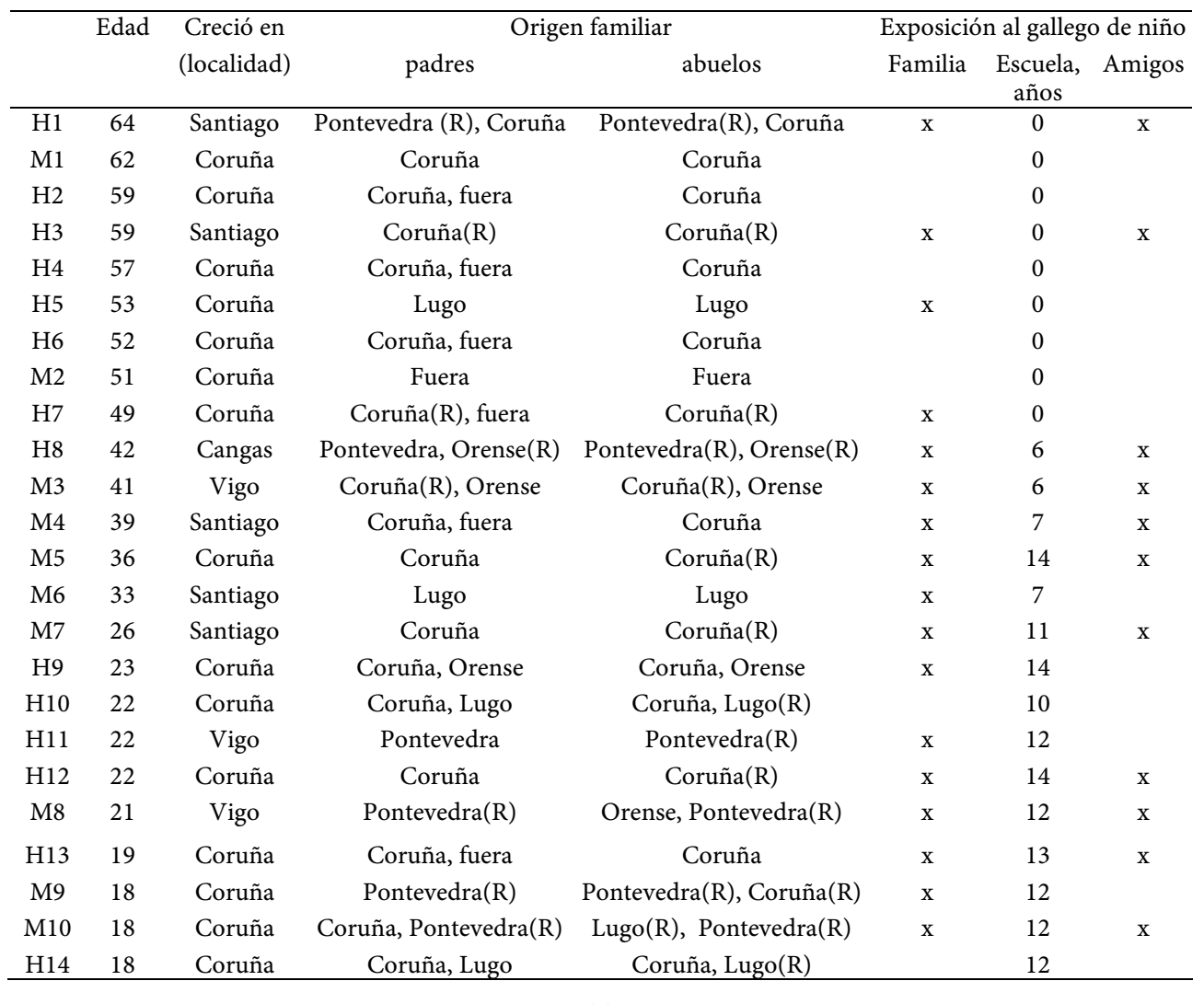

Tabla 1

\subsection{Contornos observados en la encuesta de situaciones}

Las configuraciones tonémicas que los informantes produjeron durante esta tarea fueron dos de subida, $\mathrm{L}^{\star} \mathrm{H} \%$ y $\mathrm{L}+\mathrm{H}^{\star} \mathrm{H} \%$, una circunfleja, $\mathrm{L}+\mathrm{H}^{\star} \mathrm{HL} \%$, y otra de bajada, $\mathrm{H}+\mathrm{L}^{\star} \mathrm{L} \%$. Los criterios de transcripción utilizados son los de Estebas y Prieto (2009) y los materiales de entrenamiento del Sp_ToBI recopilados por Aguilar, De la Mota y Prieto (2009). Las Figuras 2 y 3 reproducen de forma esquemática los contornos entonacionales y la notación que se asignó a cada uno. El espacio sombreado corresponde a la última sílaba tónica de la oración. 


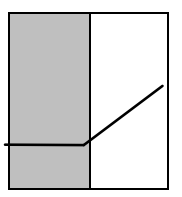

$\mathrm{L}^{*} \mathrm{H} \%$ o

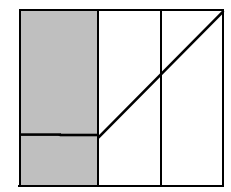

$\mathrm{L}^{\star} \mathrm{H} \%$ y

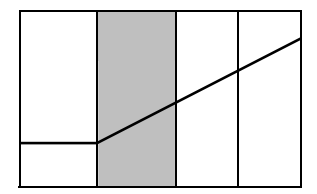

$\mathrm{L}+\mathrm{H}^{\star} \mathrm{H} \%$

Figura 2. Tonemas ascendentes en la muestra

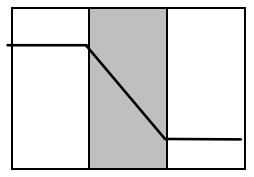

$\mathrm{H}+\mathrm{L}^{\star} \mathrm{L} \%$ y

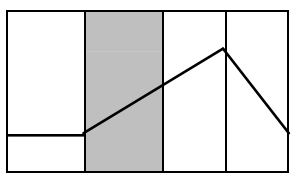

$\mathrm{L}+\mathrm{H}^{\star} \mathrm{HL} \%$

Figura 3. Tonemas descendente y circunflejo en la muestra

En la configuración $\mathrm{L}^{\star} \mathrm{H} \%$, la F0 permanece baja a lo largo de la sílaba tónica asociada con el acento nuclear y sube ya en el material postónico. En el caso de $\mathrm{L}+\mathrm{H}^{*} \mathrm{H} \%$, la subida se inicia en el ataque o hacia el principio de la sílaba nuclear. En ocasiones, el tono de frontera ascendente presenta realizaciones en las que la F0 sube bastante por encima del primer pico de la oración, contorno que se ha transcrito como $\mathrm{HH} \%$ en otros estudios sobre interrogativas en español (Estebas y Prieto, 2009; Prieto y Roseano, 2010). En su corpus del habla de Madrid, Estebas y Prieto (2010) observan que la configuración $\mathrm{L}^{\star} \mathrm{HH} \%$ ocurre en preguntas en las que se pide información, mientras que $\mathrm{L}^{\star} \mathrm{H} \%$ es una de las configuraciones posibles para las preguntas confirmatorias (Estebas y Prieto, 2010: 27-29). Este contraste no se encuentra en los datos semi-espontáneos que se obtuvieron para el presente estudio con la misma metodología empleada por Estebas y Prieto (2010). Este hecho, unido a la falta de un criterio claro para distinguir entre $\mathrm{H} \%$ y HH\% en la transcripción, es decir, cuánto debe subir la F0 para considerarse HH\%, proponemos analizar por el momento ambas realizaciones como $\mathrm{H} \%$, dejando abierta la posibilidad de recodificación si se encontrara un contraste entre ambos tonemas de subida en el castellano de Galicia. La Figura 4 ilustra uno de los tonemas ascendentes en la muestra.

En la configuración circunfleja $\mathrm{L}+\mathrm{H}^{\star} \mathrm{HL} \%$, la subida de la $\mathrm{F} 0$ empieza en el ataque de la última sílaba tónica y sigue subiendo durante el material postónico para después caer de nuevo. En el sistema de transcripción prosódica del castellano Sp_ToBI (Aguilar, De la Mota y Prieto, 2009) este tonema se interpreta como la melodía final de los vocativos o de peticiones insistentes (ej., ¿verdad que vendrás?). Recordemos que, en 
gallego, esta configuración se ha reportado para las interrogativas absolutas de hablantes de la comarca del Bajo Miño, Pontevedra (Fernández Rei, 2007).

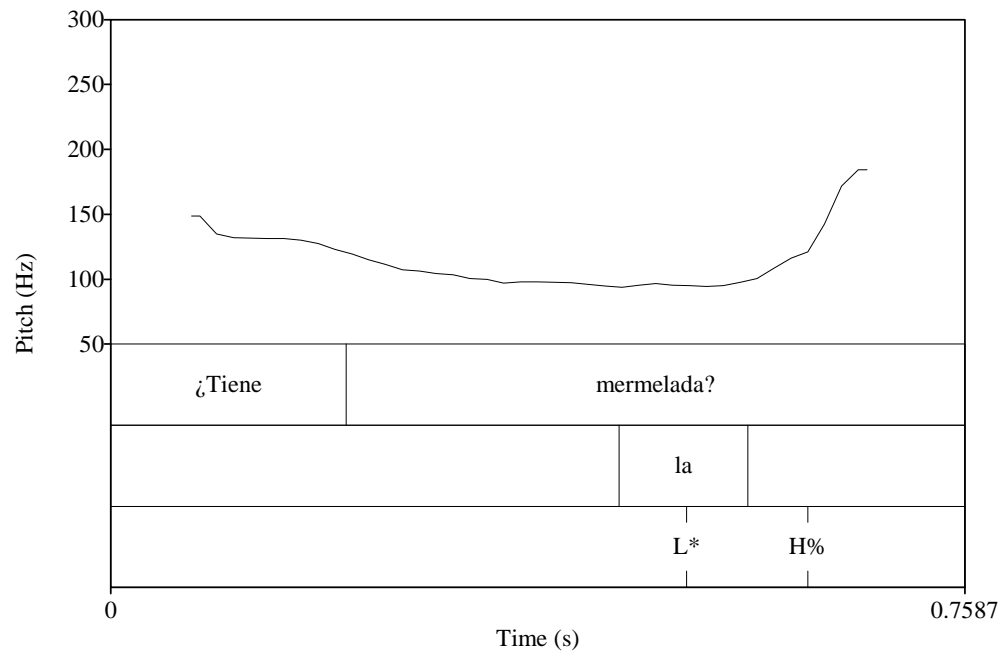

Figura 4. Respuesta a la situación núm. 18, hablante H4

En la configuración $\mathrm{H}+\mathrm{L}^{*} \mathrm{~L} \%$, el contorno de la F0 se mantiene alto desde el primer pico de la oración y comienza a bajar al principio de la sílaba nuclear. El nivel bajo se mantiene durante todo el material postnuclear. Este tonema se ha reportado para el habla de Madrid en oraciones interrogativas exhortativas (ej., ¿Queréis callar?) y antiexpectativas (ej., ¿tienes frío? en un contexto en el que hace calor) (Aguilar, De la Mota y Prieto, 2009). También se ha documentado en las interrogativas absolutas con función informativa en gallego, en particular para hablantes de Camelle y Carballo, ambas localidades al noroeste de la región (Fernández Rei, 2007). La Figura 5 ilustra el tonema descendente para un contexto de pregunta informativa en la presente muestra.

Recordemos que la encuesta de situaciones tenía como objetivo determinar si el contraste observado en otros dialectos entre el tonema ascendente y el descendente en interrogativas absolutas con distinta función pragmática tiene validez en el castellano de Galicia. Como dijimos, esta parte del estudio replica la metodología que utilizaron Estabas y Prieto (2010) y López Bobo y Cuevas Alonso (2010) para obtener enunciados semi-espontáneos en el habla de Madrid y de Cantabria respectivamente. La Tabla 2 presenta las configuraciones encontradas en sendos estudios en las interrogativas correspondientes a los tres contextos pragmáticos: preguntas informativas, preguntas de confirmación y preguntas imperativas (véase Apéndice B para los contextos específi$\cos )$. 


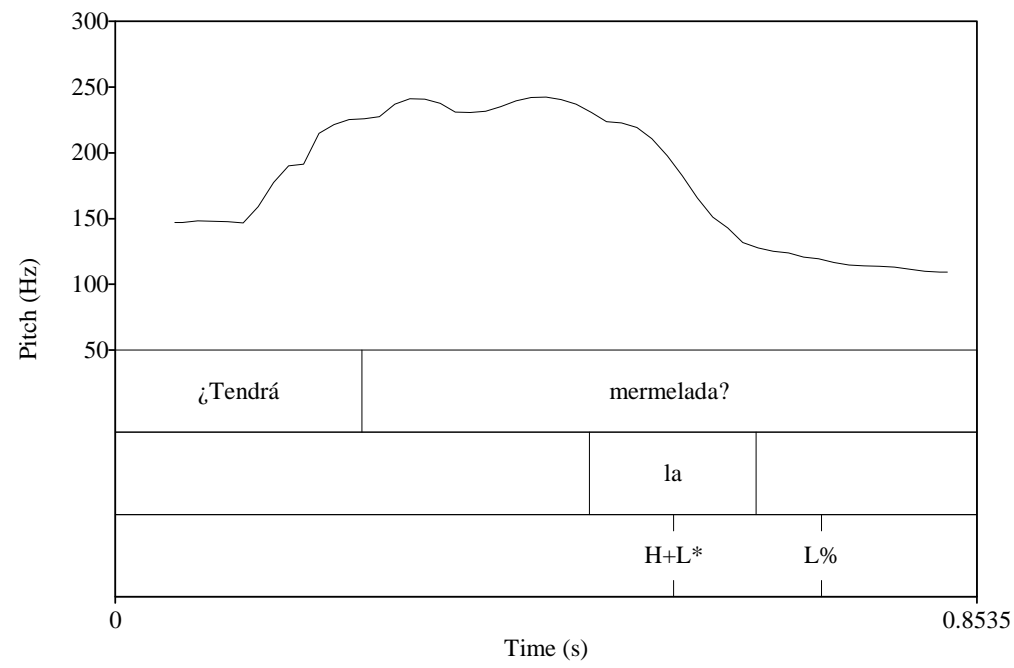

Figura 5. Respuesta a la situación núm.18, hablante H3

Vemos en la Tabla 2 que en Madrid hay un claro contraste entre el tonema descendente con el que se producen las interrogativas absolutas de confirmación o imperativas y el tonema ascendente con el que se enuncian las preguntas informativas. En Cantabria, sin embargo, se observa una variación en las preguntas informativas: pueden presentar un tonema ascendente o un tonema circunflejo que López Bobo y Cuevas Alonso (2010) vinculan a la presencia de otras variedades romances además del castellano en esta región.

\begin{tabular}{lll}
\hline & Madrid & Cantabria \\
\hline $\begin{array}{l}\text { Contexto neutro } \\
\text { pregunta informativa }\end{array}$ & $\mathrm{L}^{*} \mathrm{HH} \%$ & $\mathrm{~L}^{*} \mathrm{HH} \%$ ó $\mathrm{H}^{*} \mathrm{HL} \%$ (en la variedad tradicional) \\
\hline $\begin{array}{ll}\text { Contexto no neutro } \\
\text { pregunta de confirmación }\end{array}$ & $\mathrm{H}+\mathrm{L}^{*} \mathrm{~L} \%$ ó $\mathrm{L}^{*} \mathrm{H} \%$ & $\mathrm{~L}+\mathrm{H}^{*} \mathrm{HL} \%$ \\
pregunta imperativa & $\mathrm{H}+\mathrm{L}^{*} \mathrm{~L} \%$ & $\mathrm{H}+\mathrm{L}^{*} \mathrm{M} \%$ ó $\mathrm{L}+{ }_{\mathrm{i}} \mathrm{H}^{*} \mathrm{ML} \%$ \\
\hline
\end{tabular}

Tabla 2. Tonemas de interrogativas absolutas según función pragmática en Madrid y Cantabria

Al replicar la misma metodología con castellanohablantes de Galicia en nuestro estudio, observamos una variación similar a la de Cantabria: el contraste entre interrogativas absolutas neutras y no neutras no parece expresarse por medio de configuraciones tonémicas diferentes. La Tabla 3 detalla los contornos que cada participante produjo 
durante la encuesta de situaciones. Para cada participante se incluyen el número total de interrogativas que produjo en cada una de las tres funciones pragmáticas $(\mathrm{N})$ y la frecuencia con que los distintos contornos entonativos aparecieron en cada una (el número entre paréntesis) $)^{9}$.

Dos resultados a destacar en la Tabla 3 son: 1) para la mayoría de los hablantes de este corpus no se da el contraste pragmático entre $\mathrm{L}^{\star} \mathrm{H} \%$ y $\mathrm{H}+\mathrm{L}^{\star} \mathrm{L} \%$ observado para estos mismos contextos en Madrid y 2) aquellos hablantes que sí tienden a usar más los tonemas ascendentes en las preguntas informativas son aquellos cuyos familiares no son originarios de Galicia o, si lo son, provienen de zonas urbanas y, por lo tanto, son menos propensos a usar gallego en la casa. Este resultado sugiere un cambio de función pragmática del tonema descendente en las oraciones interrogativas absolutas del castellano de Galicia cuyo origen puede atribuirse al contacto histórico entre gallego y castellano en esta comunidad autónoma.

\begin{tabular}{|c|c|c|c|c|c|c|}
\hline & \multicolumn{2}{|c|}{$\begin{array}{l}\text { Tipo de pregunta } \\
\text { informativa }\end{array}$} & \multicolumn{2}{|c|}{ de confirmación } & \multicolumn{2}{|c|}{ imperativa } \\
\hline & $\mathrm{N}$ & & $\mathrm{N}$ & & $\mathrm{N}$ & \\
\hline H1 & 4 & $\mathrm{H}+\mathrm{L}^{\star} \mathrm{L} \%(4)$ & 2 & $\mathrm{H}+\mathrm{L}^{\star} \mathrm{L} \%(1), \mathrm{L}+\mathrm{H}^{*} \mathrm{H} \%(1)$ & 2 & $\mathrm{H}+\mathrm{L}^{\star} \mathrm{L} \%(1), \mathrm{L}+\mathrm{H}^{\star} \mathrm{HL} \%$ (1) \\
\hline M1 & 4 & $\mathrm{~L}^{\star} \mathrm{H} \%$ (3), $\mathrm{H}+\mathrm{L}^{\star} \mathrm{L} \%$ (1) & 3 & $\mathrm{~L}^{\star} \mathrm{H} \%(2), \mathrm{H}+\mathrm{L}^{\star} \mathrm{L} \%$ (1) & 5 & $\mathrm{~L}^{*} \mathrm{H} \%(5)$ \\
\hline $\mathrm{H} 2$ & 4 & $\mathrm{~L}^{\star} \mathrm{H} \%(4)$ & & -- & & -- \\
\hline $\mathrm{H} 3$ & 5 & $\mathrm{H}+\mathrm{L}^{*} \mathrm{~L} \%(5)$ & 2 & $\mathrm{H}+\mathrm{L}^{\star} \mathrm{L} \%(2)$ & 4 & $\mathrm{H}+\mathrm{L}^{*} \mathrm{~L} \%(3), \mathrm{L}^{*} \mathrm{H} \%$ (1) \\
\hline $\mathrm{H} 4$ & 4 & $\mathrm{~L}^{\star} \mathrm{H} \%(4)$ & 2 & $\mathrm{H}+\mathrm{L}^{\star} \mathrm{L} \%(2)$ & & -- \\
\hline H5 & 2 & $\mathrm{~L}^{\star} \mathrm{H} \%(2)$ & & -- & 2 & $\mathrm{~L}^{\star} \mathrm{H} \%(1), \mathrm{H}+\mathrm{L}^{\star} \mathrm{L} \%(1)$ \\
\hline H6 & 5 & $\mathrm{~L}^{\star} \mathrm{H} \%(4), \mathrm{L}+\mathrm{H}^{\star} \mathrm{H} \%$ (1) & 4 & $\mathrm{H}+\mathrm{L}^{\star} \mathrm{L} \%(4)$ & 5 & $\mathrm{H}+\mathrm{L}^{\star} \mathrm{L} \%(5)$ \\
\hline M2 & 3 & $\mathrm{~L}^{\star} \mathrm{H} \%(1), \mathrm{H}+\mathrm{L}^{\star} \mathrm{L} \%$ (2) & 1 & $\mathrm{H}+\mathrm{L}^{\star} \mathrm{L} \%(1)$ & & -- \\
\hline $\mathrm{H} 7$ & 5 & $\begin{array}{c}\mathrm{L}^{*} \mathrm{H} \%(1), \mathrm{L}+\mathrm{H}^{\star} \mathrm{H} \%(1) \\
\mathrm{H}+\mathrm{L}^{*} \mathrm{~L} \%(3)\end{array}$ & 2 & $\mathrm{H}+\mathrm{L}^{\star} \mathrm{L} \%(2)$ & 6 & $H+L^{\star} \mathrm{L} \%(6)$ \\
\hline $\mathrm{H} 8$ & 4 & $\mathrm{H}+\mathrm{L}^{*} \mathrm{~L} \%(4)$ & 3 & $\mathrm{H}+\mathrm{L}^{\star} \mathrm{L} \%(3)$ & 4 & $\mathrm{H}+\mathrm{L}^{\star} \mathrm{L} \%(4)$ \\
\hline M3 & 4 & $\mathrm{~L}^{\star} \mathrm{H} \%(1), \mathrm{H}+\mathrm{L}^{\star} \mathrm{L} \%$ (3) & 3 & $\mathrm{H}+\mathrm{L}^{\star} \mathrm{L} \%(3)$ & 3 & $\mathrm{~L}+\mathrm{H}^{\star} \mathrm{L} \%(1), \mathrm{H}+\mathrm{L}^{\star} \mathrm{L} \%$ (2) \\
\hline M4 & 5 & $\mathrm{~L}^{\star} \mathrm{H} \%(3), \mathrm{H}+\mathrm{L}^{\star} \mathrm{L} \%(2)$ & 4 & $\mathrm{~L}^{\star} \mathrm{H} \%(4)$ & 3 & $\mathrm{~L}^{\star} \mathrm{H} \%(3)$ \\
\hline
\end{tabular}

\footnotetext{
${ }^{9}$ Como ya se explicó, la disparidad en el número de producciones por informante se debe a que no todos respondieron a cada contexto con el tipo de oración deseada. Por ejemplo, hubo ocasiones en que se produjo una pregunta pronominal (ej., ¿qué hora es? en lugar de ¿tienes hora?) para el contexto núm. 19 (Apéndice B). Las interrogativas pronominales se realizan típicamente con un tonema descendente $\mathrm{H}+\mathrm{L}^{\star} \mathrm{L} \%$ en el español europeo, por lo que el posible efecto del gallego no se puede determinar en estas oraciones. Por consiguiente, ninguna interrogativa pronominal se tuvo en cuenta para el recuento.
} 


\begin{tabular}{|c|c|c|c|c|c|c|}
\hline M5 & 4 & $\mathrm{~L}^{\star} \mathrm{H} \%(2), \mathrm{H}+\mathrm{L}^{\star} \mathrm{L} \%$ (2) & 3 & $\mathrm{~L}^{\star} \mathrm{H} \%(2), \mathrm{H}+\mathrm{L}^{\star} \mathrm{L} \%$ (1) & 2 & $\mathrm{~L}^{\star} \mathrm{H} \%(1), \mathrm{H}+\mathrm{L}^{\star} \mathrm{L} \%$ (1) \\
\hline M6 & 5 & $\mathrm{H}+\mathrm{L}^{\star} \mathrm{L} \%(5)$ & 3 & $\mathrm{~L}^{\star} \mathrm{H} \%(2), \mathrm{H}+\mathrm{L}^{\star} \mathrm{L} \%$ (1) & 3 & $\mathrm{~L}^{\star} \mathrm{H} \%(2), \mathrm{H}+\mathrm{L}^{\star} \mathrm{L} \%$ (1) \\
\hline M7 & 4 & $\mathrm{~L}^{\star} \mathrm{H} \%(1), \mathrm{H}+\mathrm{L} * \mathrm{~L} \%$ (3) & 2 & $\mathrm{H}+\mathrm{L}^{\star} \mathrm{L} \%(2)$ & 5 & $\mathrm{~L}^{\star} \mathrm{H} \%(1), \mathrm{H}+\mathrm{L}^{\star} \mathrm{L} \%$ (4) \\
\hline H9 & 5 & $\mathrm{~L}^{*} \mathrm{H} \%(2), \mathrm{L}+\mathrm{H}^{*} \mathrm{H} \%$ (3) & 2 & $\mathrm{~L}+\mathrm{H}^{\star} \mathrm{H} \%(2)$ & 2 & $\mathrm{H}+\mathrm{L}^{\star} \mathrm{L} \%(2)$ \\
\hline $\mathrm{H} 10$ & 5 & $\mathrm{~L}+\mathrm{H}^{\star} \mathrm{H} \%(2), \mathrm{H}+\mathrm{L}^{*} \mathrm{~L} \%$ & 3 & $\mathrm{H}+\mathrm{L}^{\star} \mathrm{L} \%(3)$ & 4 & $\mathrm{H}+\mathrm{L}^{\star} \mathrm{L} \%(4)$ \\
\hline H11 & 5 & $\mathrm{~L}^{\star} \mathrm{H} \%(1), \mathrm{H}+\mathrm{L} * \mathrm{~L} \%$ & 2 & $\mathrm{H}+\mathrm{L}^{\star} \mathrm{L} \%(2)$ & 4 & $\mathrm{H}+\mathrm{L}^{\star} \mathrm{L} \%(4)$ \\
\hline H12 & 5 & $\mathrm{~L}+\mathrm{H}^{\star} \mathrm{HL} \%(1), \mathrm{H}+\mathrm{L}^{\star} \mathrm{L} \%$ (4) & 3 & $\mathrm{H}+\mathrm{L}^{\star} \mathrm{L} \%(3)$ & 2 & $\mathrm{H}+\mathrm{L}^{\star} \mathrm{L} \%(2)$ \\
\hline M8 & 4 & $\mathrm{H}+\mathrm{L}^{\star} \mathrm{L} \%(4)$ & 2 & $\mathrm{H}+\mathrm{L}^{\star} \mathrm{L} \%(2)$ & 3 & $\mathrm{H}+\mathrm{L}^{\star} \mathrm{L} \%$ \\
\hline H13 & 5 & $\mathrm{~L}^{*} \mathrm{H} \%(1), \mathrm{H}+\mathrm{L}^{\star} \mathrm{L} \%$ (4) & 2 & $\mathrm{H}+\mathrm{L}^{\star} \mathrm{L} \%(2)$ & 4 & $\mathrm{H}+\mathrm{L}^{\star} \mathrm{L} \%(4)$ \\
\hline M9 & 5 & $\mathrm{~L}+\mathrm{H}^{\star} \mathrm{HL} \%(1), \mathrm{H}+\mathrm{L}^{\star} \mathrm{L} \%$ (4) & 4 & $\mathrm{H}+\mathrm{L}^{\star} \mathrm{L} \%(4)$ & 4 & $\mathrm{~L}^{\star} \mathrm{H} \%(2), \mathrm{H}+\mathrm{L}^{\star} \mathrm{L} \%$ (2) \\
\hline M10 & 5 & $\mathrm{H}+\mathrm{L}^{\star} \mathrm{L} \%(5)$ & 4 & $\mathrm{H}+\mathrm{L}^{\star} \mathrm{L} \%(4)$ & 5 & $\mathrm{H}+\mathrm{L}^{\star} \mathrm{L} \%(5)$ \\
\hline H14 & 5 & $\mathrm{H}+\mathrm{L}^{\star} \mathrm{L} \%(5)$ & 3 & $\mathrm{H}+\mathrm{L}^{\star} \mathrm{L} \%$ & 3 & $\mathrm{H}+\mathrm{L}^{\star} \mathrm{L} \%$ \\
\hline
\end{tabular}

Tabla 3. Tonemas producidos en la encuesta de situaciones

La encuesta de situaciones tiene la ventaja metodológica de provocar enunciados más espontáneos que la lectura de frases, pero cuenta con el inconveniente de que no todos los hablantes produjeron enunciados idénticos para todos los contextos. Este hecho puede limitar un análisis estadístico de los factores sociales asociados con la variación observada en esta tarea, ya que no podríamos descartar que las diferencias se debieran a motivos estructurales (i.e., número de sílabas, posición de las sílabas tónicas, etc.). La lectura de frases, sin embargo, pese a producir un habla más formal, sí que nos permite controlar la estructura de los enunciados. A continuación se reportan los resultados de la lectura de frases y del análisis de regresión logística que examina los factores sociales que inciden en el uso de tonemas descendentes en preguntas informativas.

\subsection{Contornos observados en la lectura de frases}

Con el fin de controlar la posibilidad de variación por contexto pragmático, la lectura de frases se diseñó para provocar solo preguntas en un contexto neutro de petición de información. Tras descartar los errores (titubeos o pausas), se transcribieron un total de 1145 interrogativas absolutas siguiendo las pautas del sistema Sp_ToBI recopiladas por Aguilar, De la Mota y Prieto (2009), salvo en el análisis del tono de frontera ascendente $\mathrm{H} \%$, como se explicó anteriormente. De la muestra se extrajeron las cuatro configuraciones tonémicas presentes también en la encuesta de situaciones. Hubo hablantes que solo produjeron uno de los contornos y otros que alternaron configuraciones, en ocasiones incluso para la misma oración dentro del mismo contexto (i.e., repitieron cada enunciado dos veces con contornos diferentes cada vez). La Figura 6 muestra un 
ejemplo de este tipo de variación. 6a corresponde a la primera repetición de la frase y 6b a la segunda repetición del mismo estímulo.

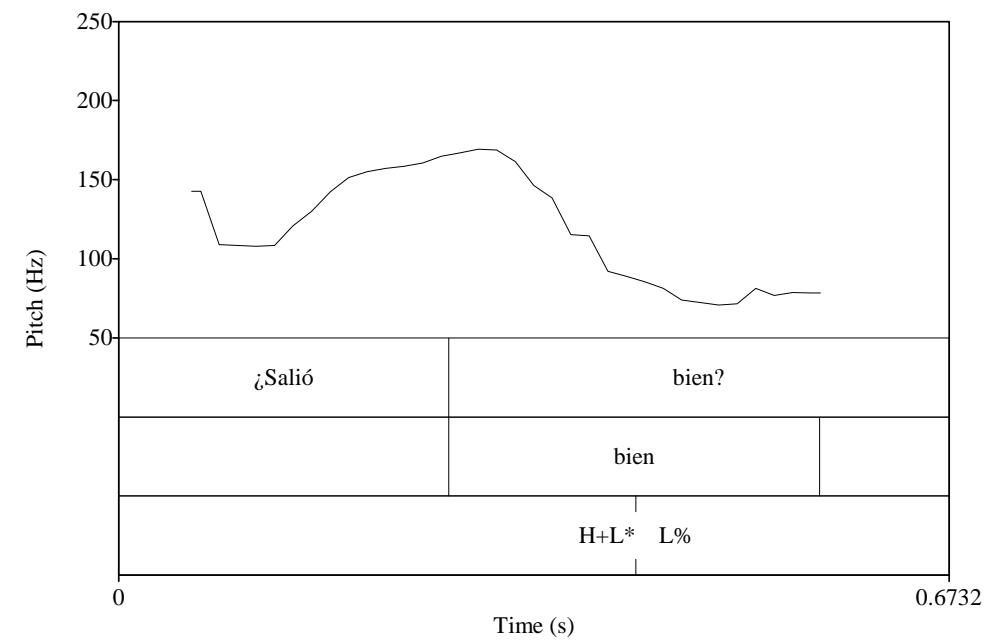

Figura 6a. Contexto Estás hablando con Jorge y quieres saber cómo le fue en un examen. Preguntas: ¿Salió bien? Primera repetición del hablante H8

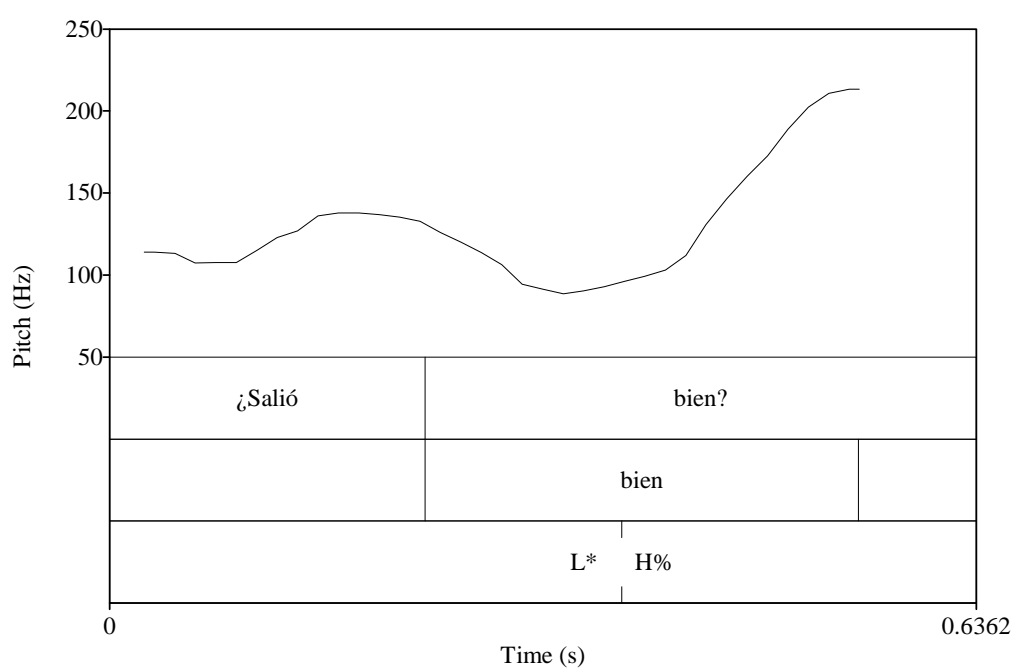

Figura 6b. Contexto Estás hablando con Jorge y quieres saber cómo le fue en un examen. Preguntas: ¿Salió bien? Segunda repetición del hablante H8

La incidencia de cada configuración tonémica en la lectura de frases de cada participante se calculó dividiendo el número de veces que un individuo presentaba un deter- 
minado patrón por el número total de interrogativas producidas por dicho individuo. La Tabla 4 presenta los porcentajes de cada configuración para cada participante.

\begin{tabular}{|c|c|c|c|c|c|c|c|c|c|}
\hline \multirow[t]{2}{*}{ Hablante } & \multicolumn{2}{|c|}{$\mathbf{H}+\mathbf{L}^{\star} \mathbf{L} \%$} & \multicolumn{2}{|c|}{$\mathbf{L}+\mathbf{H}^{*} \mathbf{H L} \%$} & \multirow{2}{*}{$\begin{array}{c}\mathbf{L}^{*} \mathbf{H} \% \\
\mathrm{~N} \\
\end{array}$} & \multicolumn{3}{|c|}{$\mathbf{L}+\mathbf{H}^{\star} \mathbf{H} \%$} & \multirow{2}{*}{$\begin{array}{r}\text { Total } \\
\mathrm{N}\end{array}$} \\
\hline & $\mathrm{N}$ & $\%$ & $\mathrm{~N}$ & $\%$ & & $\%$ & $\mathrm{~N}$ & $\%$ & \\
\hline $\mathrm{H} 1$ & & & 48 & $100 \%$ & & & & & 48 \\
\hline M1 & & & & & 48 & $100 \%$ & & & 48 \\
\hline $\mathrm{H} 2$ & & & & & 40 & $83.3 \%$ & 8 & 16.7 & 48 \\
\hline H3 & 28 & $59.5 \%$ & & & 19 & $40.5 \%$ & & & 47 \\
\hline $\mathrm{H} 4$ & & & & & 48 & $100 \%$ & & & 48 \\
\hline H5 & & & & & 48 & $100 \%$ & & & 48 \\
\hline H6 & & & & & 48 & $100 \%$ & & & 48 \\
\hline M2 & & & & & 46 & $95.8 \%$ & 2 & $4.2 \%$ & 48 \\
\hline $\mathrm{H} 7$ & & & & & 48 & $100 \%$ & & & 48 \\
\hline $\mathrm{H} 8$ & 6 & $13 \%$ & & & 40 & $87 \%$ & & & 46 \\
\hline M3 & & & & & 23 & $48 \%$ & 25 & $52 \%$ & 48 \\
\hline M4 & & & & & 48 & $100 \%$ & & & 48 \\
\hline M5 & & & & & 48 & $100 \%$ & & & 48 \\
\hline M6 & & & & & 48 & $100 \%$ & & & 48 \\
\hline M7 & & & & & 48 & $100 \%$ & & & 48 \\
\hline H9 & & & & & 36 & $76.6 \%$ & 11 & $23.4 \%$ & 47 \\
\hline $\mathrm{H} 10$ & 32 & $66.6 \%$ & & & 8 & $16.7 \%$ & 8 & $16.7 \%$ & 48 \\
\hline H11 & 4 & $8.5 \%$ & & & 35 & $74.5 \%$ & 8 & $17 \%$ & 47 \\
\hline H12 & & & 8 & 16.7 & 31 & $64.6 \%$ & 9 & $18.7 \%$ & 48 \\
\hline M8 & 11 & $23 \%$ & & & 32 & $66.6 \%$ & 5 & $10.4 \%$ & 48 \\
\hline H13 & & & & & 36 & $75 \%$ & 12 & $25 \%$ & 48 \\
\hline M9 & & & 25 & 52 & 23 & $33.4 \%$ & 7 & $14.6 \%$ & 48 \\
\hline M10 & 45 & $94 \%$ & & & 3 & $6 \%$ & & & 48 \\
\hline H14 & & & & & 46 & $100 \%$ & & & 46 \\
\hline
\end{tabular}

Tabla 4. Incidencia de cada tonema en la tarea de lectura

Al contrastar los resultados de las Tablas 3 y 4 salta a la vista que para algunos hablantes el uso del tonema descendente en las preguntas informativas está estilísticamente motivado. Mientras que hubo hablantes que pasaron de usar un tonema descendente en las preguntas informativas de la tarea más informal a usar el tonema ascendente en la tarea formal, el movimiento en la dirección contraria no ocurrió. De la comparación de ambas tablas merece la pena destacar tres resultados:

a) En el castellano de Galicia aún se da la variación prosódico-pragmática reportada en Madrid (Estebas y Prieto, 2010), ya que los cinco hablantes (H2, H4, H5, H6, H9) que solo usaron el tonema ascendente para las preguntas informativas en la encuesta de situaciones lo mantuvieron en la tarea de lectura.

b) Para los hablantes más expuestos al gallego o a un castellano más agallegado, la elección entre un tonema ascendente o uno descendente en las preguntas informativas parece seguir criterios estilísticos: ocho hablantes (M1, M2, M3, M5, M4, M7, H7 y H13) que utilizaron tanto el tonema ascendente como el descendente y dos (M6 y H14) que solo utilizaron el tonema descendente en las 
preguntas informativas de la tarea semi-espontánea cambiaron para utilizar el tonema ascendente de forma categórica en la tarea más formal. De estos diez, siete son mujeres, lo cual sugiere que esta elección puede estar sujeta a factores sociales además de estilísticos.

c) La diversidad geográfica reportada para los contornos entonacionales de las interrogativas absolutas en gallego también aparece en el castellano de esta región. Aunque la mayoría de los hablantes alternaron entre $\mathrm{L}^{\star} \mathrm{H} \%$ y $\mathrm{H}+\mathrm{L}^{\star} \mathrm{L} \%$, hubo tres (H1, M9 y H12) que usaron también el tonema circunflejo $\mathrm{L}+\mathrm{H}^{\star} \mathrm{HL} \%$ reportado en las interrogativas absolutas de la comarca de Bajo Miño (Fernández Rei, 2007), en la provincia de Pontevedra. Al volver a los datos del cuestionario sociolingüístico (véase la Tabla 1) constatamos que los tres hablantes que usaron este tonema tienen familia de la provincia de Pontevedra. Este dato apoya la hipótesis del influjo gallego en la entonación del castellano de Galicia.

El tipo de variación observada indica que las configuraciones ascendentes contrastan fonológicamente con la descendente y la circunfleja para los hablantes menos expuestos al gallego vernáculo. Para el resto de los hablantes, las configuraciones observadas en la muestra parecen comportarse como variantes de la entonación interrogativa absoluta para el castellano de Galicia y la incidencia de cada patrón está motivada por factores extralingüísticos relacionados con el bilingüismo de la región. Aunque las cuatro configuraciones no parecen contrastar fonológicamente entre sí para la mayoría de los individuos en la muestra, asumimos que sí pueden servir para diferenciar las interrogativas de otros tipos de oraciones (ej. declarativas), pero la constatación de tal propuesta queda fuera del alcance de este trabajo.

Puesto que hay hablantes que no presentan variación, se podría decir que para aquellos que sí la presentan ésta se debe explicar por factores extralingüísticos más que lingüísticos. Con el fin de determinar qué factores favorecen los tonos de frontera descendente o circunflejo frente al ascendente cuando el contexto pragmático es el mismo (i.e., pregunta informativa), se realizó un análisis de regresión logística (GoldVarb) de las interrogativas $(\mathrm{N}=380)$ de los ocho hablantes que presentaban variación en la tarea de lectura. Los resultados de dicho análisis se reportan a continuación.

\subsection{Factores extralingüísticos que favorecen $\mathrm{H}+\mathrm{L}^{\star} \mathrm{L} \% \mathrm{o} \mathrm{L}+\mathrm{H}^{\star} \mathrm{HL} \%$ entre los hablantes con variación}

El análisis de regresión logística modela la probabilidad de un evento en función de una serie de variables explicativas. La contribución a la variación de cada categoría de las variables explicativas se estima de tal forma que un factor con peso mayor de .5 se 
considera que favorece una variante particular. La fuerza relativa de cada variable independiente se determina por el rango: cuanto mayor sea éste para una variable, mayor es su magnitud de efecto. Los grupos de factores que se tuvieron en cuenta en el análisis fueron la edad, el género, el tipo de exposición al gallego en casa cuando eran pequeños, la localidad donde crecieron y si el enunciado había sido la primera o la segunda repetición de la oración interrogativa ${ }^{10}$.

\subsubsection{Variables independientes}

En la exploración preliminar de los datos se observó una fuerte correlación entre edad y años de instrucción formal en gallego. Como muestra la Tabla 1, los individuos más jóvenes de la muestra tuvieron exposición al gallego en la escuela desde una edad temprana como resultado de los cambios en política lingüística impulsados por el Estatuto Autonómico de Galicia de 1981. Dada la fuerte correlación entre edad e instrucción en gallego, lo más apropiado para el tipo de análisis estadístico escogido en esta investigación es combinar ambas variables en una sola que capture la naturaleza de dicha interacción. Esto se hizo separando la edad en dos niveles generacionales: los nacidos antes de 1975 y los nacidos este año o después. La motivación de esta fecha de corte es que, teniendo en cuenta cuándo entraron en vigor los cambios en política lingüística en Galicia, cualquier individuo nacido en 1975 o después empezó a aprender gallego a una edad suficientemente temprana - al menos 6 años - para adquirirla de una forma casi nativa. El factor de la edad, por tanto, se puede considerar en este análisis como una medida del efecto generacional en la variación y a la vez del efecto de haber recibo instrucción formal en gallego.

En cuanto a la exposición al gallego en la familia durante la niñez, recordemos que el cuestionario sociolingüístico preguntaba a los informantes si habían recibido input en gallego de sus padres y/o de sus abuelos. De nuevo el análisis preliminar de ambas variables demostró una interacción tal que, si los padres usaban el gallego en casa, probablemente los abuelos también lo hicieran. Por tanto, se decidió considerar solo el input recibido de los progenitores para los propósitos del análisis estadístico. Aunque el cuestionario sociolingüístico pedía que se evaluara la cantidad de exposición al gallego en distintos ambientes sobre una escala de cero a diez, el análisis de regresión con Gold-

\footnotetext{
${ }^{10}$ Como dijimos, teniendo en cuenta la asunción de que la segunda repetición de una forma lingüística es más cuidada o formal que la primera (Labov, 1972), el examen del orden de la repetición como variable manipulada puede esclarecer la estratificación estilística de la entonación interrogativa en el castellano de Galicia.
} 
varb requiere variables independientes nominales. Es por ello que las respuestas al cuestionario se reinterpretaron del siguiente modo: una respuesta de cero equivale a ninguna exposición y cualquier respuesta de 1 a 10 equivale a alguna exposición. Es una limitación de la metodología no poder considerar el grado de exposición al gallego como variable continua, pero los valores individuales reportados por cada participante todavía resultan útiles para la interpretación cualitativa de las tendencias observadas en el análisis cuantitativo. Los niveles del factor de exposición al gallego en la familia fueron: uso del gallego por los dos padres, por uno solo de los padres y por ninguno de los padres. Teniendo en cuenta las investigaciones recientes sobre la influencia de la madre en el desarrollo etnolingüístico de un individuo (Potowski, 2008) ${ }^{11}$ el nivel solo un padre se sub-dividió en solo la madre o solo el padre.

En cuanto a la variable de localidad donde el informante creció, el reducido tamaño de la muestra impidió que este factor tuviera más de dos niveles. El hecho de que La Coruña se considere una población tradicionalmente más castellanohablante que las otras ciudades en la muestra justifica una división entre los que crecieron en La Coruña y los que no (que combinaba Vigo, Santiago y un individuo de Cangas, cerca de Vigo).

\subsubsection{Variable dependiente y resultados}

Las categorías de la variable de respuesta fueron la presencia del tono de frontera ascendente $\mathrm{H} \%$ frente a su ausencia. Se considera que un factor favorece $\mathrm{H} \%$ cuando es mayor de .5, mientras que un factor con peso menor de .5 favorece la ausencia del tono $\mathrm{H} \%$, es decir, favorece L\% o HL\%, que se asocian con las interrogativas absolutas neutras en gallego. Al hacer el primer recorrido del análisis binomial, el género del hablante y el orden de la repetición de cada estímulo resultaron no ser estadísticamente significativos. Por tanto, se excluyeron de la segunda fase del análisis que determinó el peso relativo de cada nivel de los tres grupos de factores significativos. La Tabla 5 muestra los resultados del análisis.

El reducido valor de $\mathrm{X}^{2}$ total y por celda, ambos por debajo de 1 , significa que los factores no interactúan y que el modelo es apropiado para explicar la variación observada en esta parte del estudio. Los factores que favorecen $\mathrm{H} \%$ son aquellos cuyo peso es superior a $.5 \mathrm{y}$, por ende, los factores cuyo peso es menor de .5 favorecen L\% o HL\%, los

\footnotetext{
${ }^{11}$ En un estudio de contacto dialectal dentro de la misma unidad familiar, es decir, cuando el padre y la madre hablan dialectos diferentes de la misma lengua, Potowski (2008) encontró que en la mayoría de los casos, los informantes habían adquirido la variedad dialectal de la madre, no la del padre.
} 
dos tonos de frontera que no se usan en el castellano de Madrid en el contexto pragmático de pregunta informativa. Tales factores son, en orden de efecto en la variación (rango): que los dos padres usaran algo de gallego en casa, haber crecido en La Coruña y haber nacido antes de 1975. El primer factor, la exposición al gallego en el ambiente familiar, apoya la hipótesis de transferencia de rasgos entonacionales por contacto de lenguas que explora este trabajo. Sin embargo, dentro de este grupo se observa que el hecho de que ninguno de los dos padres hablara gallego en casa favorece $\mathrm{H} \%$, aunque solo de forma muy débil (.530). El segundo factor significativo en la variación, el área de Galicia en la que crecieron los participantes, no apoya en principio la hipótesis del contacto, puesto que el área que desfavorece el tono de frontera $\mathrm{H} \%$ en esta muestra es $\mathrm{La}$ Coruña, tradicionalmente considerada predominantemente castellanohablante. En cuanto a la edad, haber nacido antes de 1975 desfavorece la incidencia de tonos de frontera $\mathrm{H} \%$ en este análisis, mientras que haber nacido en o después de esa fecha favorece $\mathrm{H} \%$, aunque no de forma fuerte, ya que el peso de este factor solo está ligeramente por encima de .5 (peso $=.585)$. Discutiremos estos resultados en la Sección 5 teniendo en cuenta el origen de los padres y abuelos de los individuos analizados.

\begin{tabular}{|c|c|c|c|}
\hline \multicolumn{4}{|c|}{ Aplicación: presencia de H\%, N=380, Input 0.609} \\
\hline Factor & Peso & Frecuencia (apl./no apl.) & $\%$ \\
\hline \multicolumn{4}{|l|}{ Edad } \\
\hline nacido en o después de 1975 & 589 & 56 & 69 \\
\hline nacido antes de 1975 & 247 & 63 & 34 \\
\hline Rango & \multicolumn{2}{|c|}{342} & \\
\hline \multicolumn{4}{|l|}{ Exposición familiar } \\
\hline $\begin{array}{l}\text { los dos padres hablaban algo de gallego } \\
\text { en casa }\end{array}$ & 188 & 41 & 27 \\
\hline $\begin{array}{l}\text { solo el padre hablaba algo de gallego en } \\
\text { casa }\end{array}$ & 695 & 67 & 78 \\
\hline \multicolumn{2}{|c|}{$\begin{array}{l}\text { ninguno de los padres hablaba gallego en } 530 \\
\text { casa }\end{array}$} & .62 & 64 \\
\hline \multicolumn{2}{|l|}{$\begin{array}{l}\text { solo la madre hablaba algo de gallego en } 925 \\
\text { casa }\end{array}$} & 83 & 95 \\
\hline \multicolumn{2}{|l|}{ Rango } & \multicolumn{2}{|c|}{737} \\
\hline \multicolumn{4}{|l|}{ Localidad } \\
\hline no han crecido en La Coruña & 852 & 74 & 90 \\
\hline han crecido en La Coruña & 153 & 43 & 22 \\
\hline Rango & \multicolumn{2}{|c|}{699} & \\
\hline \multicolumn{4}{|l|}{ Total $\mathrm{X}^{2}=0.8755 ; \mathrm{X}^{2} /$ celda $=0.1094$} \\
\hline \multicolumn{4}{|c|}{ Log de verosimulitud (sign.) $=-186.110(<.001)$} \\
\hline
\end{tabular}

Tabla 5. Factores que favorecen los tonos $\mathrm{H} \%$ en la muestra 


\section{Discusión}

En las secciones anteriores hemos descrito las configuraciones tonémicas que presentan las interrogativas absolutas en el castellano de Galicia. El análisis estadístico de los datos de la tarea de lectura muestra que el grado de exposición al gallego dentro de la familia es el factor de mayor peso en la variación: el hecho de que ambos padres usaran el gallego en casa favorece que un individuo produzca un tonema descendente o circunflejo en preguntas informativas cuando se controla el contexto pragmático, la estructura segmental y sintáctica y el grado de formalidad de la tarea. Este dato apoya de forma robusta la hipótesis de extensión pragmática por contacto de lenguas que explora este estudio y no necesita mayor discusión en este apartado. Los resultados en cuanto a la variación por contexto pragmático, geografía y formalidad de la tarea sí que merecen más atención.

\subsection{Variación por contexto pragmático}

En la muestra hay hablantes $(\mathrm{H} 2, \mathrm{H} 4, \mathrm{H} 5, \mathrm{H} 6, \mathrm{H} 9)$ que de manera consistente usaron contornos ascendentes $\mathrm{L}^{\star} \mathrm{H} \% \mathrm{o} \mathrm{L}+\mathrm{H}{ }^{\star} \mathrm{H} \%$ al enunciar preguntas informativas tanto en la encuesta de situaciones como en la tarea más formal de lectura de frases. Cuando produjeron una configuración descendente, $\mathrm{H}+\mathrm{L}^{\star} \mathrm{L} \%$, fue en los contextos de confirmación o de pregunta imperativa, lo cual es consistente con el contraste pragmático observado en los tres tipos de interrogativas absolutas en hablantes madrileños (Estebas y Prieto, 2010). Al examinar la historia sociolingüística de estos hablantes encontramos un rasgo común: ni sus padres ni abuelos crecieron en zonas rurales de Galicia. La excepción es el hablante $\mathrm{H} 5$, cuyos abuelos son originarios de la provincia de Lugo, que es junto a Orense una de las áreas de mayor conservación del gallego según las estadísticas sobre la situación sociolingüística de Galicia (Monteagudo y Lorenzo, 2006: 54). Sin embargo, este hablante también pasó largas temporadas fuera de Galicia de pequeño, lo cual puede explicar que mantenga el contraste prosódico-pragmático del castellano central.

La relevancia de la distinción entre un origen familiar rural o urbano en Galicia radica en que los municipios de menor población son los que más han mantenido y siguen manteniendo la lengua vernácula (Monteagudo y Lorenzo, 2006: 21). Las ciudades gallegas, en cambio, han sido históricamente más resistentes a la influencia lingüística gallega, bien porque sus habitantes procedían de otras regiones lejos del dominio lingüístico gallego o bien porque los llegados de zonas rurales de Galicia pronto abandonaron el gallego como vehículo de comunicación (O’Rourke, 2006: 180). El predominio del castellano en las ciudades gallegas tuvo múltiples causas, entre ellas la formación de 
estereotipos por los que se asociaban los rasgos lingüísticos más propios del gallego con hablantes de poco nivel sociocultural y la política lingüística centralizadora del régimen franquista (1939-1975) que favorecía el uso exclusivo del castellano en los registros más altos (Ramallo, 2007). Es de esperar, por tanto, que los hablantes sin vínculos familiares en zonas rurales de Galicia tuvieran poco o ningún input en gallego o en castellano fuertemente agallegado dentro de sus círculos sociales, lo cual explica que mantengan el contraste prosódico-pragmático que se encuentra en las preguntas informativas del centro de la Península Ibérica.

\subsection{Variación por factores geográficos}

El contraste prosódico-pragmático de las interrogativas absolutas del castellano central pierde vigencia entre aquellos hablantes con mayor arraigo familiar en Galicia, hecho que se explica por la ausencia de este contraste en gallego, la lengua vernácula de la región. En la tarea semi-espontánea, la mayoría de los hablantes alternaron entre el tonema ascendente y el descendente en las preguntas informativas, pero aquellos hablantes que solo mostraron contornos más propios del gallego para este contexto pragmático comparten el hecho de que su familia más inmediata procede o de localidades muy pequeñas de Galicia o, si proceden de ciudades, estas son Orense o Lugo, los dos centros urbanos con mayor mantenimiento de la lengua vernácula como vimos en el apartado anterior. Por tanto, el origen geográfico de la familia parece tener un efecto en el grado en que los castellanohablantes de Galicia muestran patrones prosódicopragmáticos más propios del gallego que del castellano, al menos en el habla semiespontánea.

Además del grado de influencia del gallego, el origen familiar también incide en el tipo de configuraciones tonémicas que producen los individuos de nuestra muestra, ya que los tres hablantes que produjeron el tonema circunflejo $\mathrm{L}+\mathrm{H}^{\star} \mathrm{HL} \%$ documentado en las interrogativas del gallego de Bajo Miño tenían arraigo familiar en este área de Galicia. Estos resultados apoyan la hipótesis del influjo gallego en la entonación del castellano de Galicia y sugieren que dicho influjo refleja además la variación geográfica observada en la lengua gallega.

Otro dato relevante sobre la variación geográfica que revela nuestro análisis es el hecho de que entre los ocho hablantes que mostraron variación entre los tonemas ascendente, descendente y circunflejo en la tarea de lectura, el hecho de haber crecido en La Coruña se demostró estadísticamente favorable para las configuraciones más propias del gallego. Este resultado sorprende si tenemos en cuenta que La Coruña se considera una de las áreas urbanas de Galicia con menor presencia de monolingües de gallego o 
bilingües con predominio de la lengua vernácula (Monteagudo y Lorenzo, 2006: 54). Sin embargo, hay que tener en cuenta que no todos los 24 participantes del estudio se incluyeron en el análisis estadístico, sino solo los ocho que presentaron variación en la prueba de lectura. Tres de los cuatro coruñeses incluidos tienen ascendencia familiar fuera de La Coruña (véase la Tabla 1). Quizá sea por eso que estos individuos mostraron variación y pudieron ser parte del análisis estadístico, mientras que los informantes que quedaron excluidos por no mostrar variación en la lectura de frases vienen mayoritariamente de La Coruña.

Por lo tanto, aunque a primera vista los resultados del análisis del factor localidad no parezcan coincidir con las tendencias sociolingüísticas observadas a mayor escala en la población gallega, un segundo examen cualitativo más detallado del origen familiar de los participantes muestra que la influencia del gallego en los patrones prosódicopragmáticos de los castellanoparlantes de la región tiende a ser mayor si la familia de dicho individuo procede de áreas menos pobladas o menos urbanizadas. Tales áreas constituyen precisamente la localización de mayor mantenimiento del gallego.

\subsection{Variación estilística y estigmatización de lo gallego}

Las dos tareas analizadas en este estudio corresponden a distintos grados de formalidad en función de la atención que se supone que el hablante presta a la manera en que enuncia (Labov, 2001). Bajo esta asunción, la encuesta de situaciones es menos formal que la tarea de lectura de frases. Al cambiar de registro, de los 19 hablantes que no mostraron el contraste pragmático entre $\mathrm{H}+\mathrm{L}^{\star} \mathrm{L} \%$ y $\mathrm{L}^{\star} \mathrm{H} \%$ (o $\mathrm{L}+\mathrm{H}^{\star} \mathrm{H} \%$ ) típicamente castellano en el habla semi-espontánea, hubo 10 hablantes que categóricamente usaron el tonema ascendente castellano, $\mathrm{L}^{\star} \mathrm{H} \%$ (o $\mathrm{L}+\mathrm{H}^{\star} \mathrm{H} \%$ ), en el habla más formal. Recordemos que la encuesta de situaciones se administró a todos los participantes después de la lectura de frases, por lo que cualquier efecto del orden de tareas fue el mismo para toda la muestra. Esta alteración de las reglas prosódico-pragmáticas que acompaña el cambio en la formalidad de la tarea puede considerarse reflejo de "una consciente y expresa voluntad por hablar bien - o lo mejor posible - el español" (Porto Dapena, 2001, n.p.) en situaciones en las que se presta mayor atención al habla.

El uso de una pronunciación más propia del castellano estándar ${ }^{12}$ que del gallego en situaciones comunicativas más formales se ha observado también en lengua gallega.

\footnotetext{
${ }^{12}$ El habla del centro de la península es la que tradicionalmente se considera estándar nacional (Cestero, Molina y Paredes, 2008). El gallego y el castellano son lenguas tipológicamente cercanas, por lo que es muy probable que la transferencia de rasgos sea bidireccional.
} 
Castro (2003), Beswick $(2007,2010)$ y Regueira Fernández (2004) señalan que la falta de un esfuerzo normalizador del gallego oral, similar al llevado a cabo en la escritura, ha favorecido la interferencia del castellano estándar en la pronunciación de la lengua vernácula. En particular, advierten que el gallego normativo que se difunde por los medios de comunicación no toma como modelo las características orales del gallego vernáculo, pues a menudo se trata de una forma de gallego aprendida como segunda lengua por hablantes de castellano como L1. Los autores expresan alarma ante este hecho, ya que la lengua de los medios goza de prestigio social y es a menudo imitada por los jóvenes, a riesgo de perpetuar la diferenciación sociolingüística entre patrones vernáculos y formas más castellanizadas. Beswick nota también que incluso en las emisoras castellanohablantes "many of the broadcasters try to emulate a spoken Castilian that approximates a more standard form despite being bilingual" (Beswick, 2010: 41).

La tendencia a usar patrones prosódico-pragmáticos más propios del castellano que del gallego en la tarea formal del presente estudio parece relacionarse también con la estigmatización tradicional de los rasgos más agallegados. En su discusión de los efectos sociolingüísticos del contacto histórico entre gallego y español, Porto Dapena señala que "a un niño se le corrige, por ejemplo, cuando, expresándose en castellano, comete algún desliz que suene a galleguismo, y desde luego cualquiera que utilice la lengua del Estado con abundancia de elementos gallegos puede provocar fácilmente la hilaridad de sus oyentes" (Porto Dapena, 2001, n.p.). Bouzada Fernández (2003) sitúa el origen de esta estigmatización en la imagen de ruralidad con la que se asociaban los hablantes de gallego hasta bien entrado el siglo XX (pág. 325), pero añade que este estereotipo puede estar perdiendo fuerza entre los más jóvenes, socializados durante la democracia y en una época en la que el gallego está muy presente en la escuela y otras instituciones públicas. Para Bouzada Fernández, los cambios sociales experimentados en Galicia desde la llegada de la democracia contribuyen a un proceso de revalorización de lo gallego.

Social upward mobility, as a general reality, has provided the children of the neo urbanites a greater liberty to read their culture from a dignified perspective that allows them the capacity to value their own culture as an attribute rather than a stigma to ward away or dissolve (Bouzada Fernández, 2003: 327).

En la presente investigación, sin embargo, el grupo de hablantes que presentaron variación estilística incluye un número desigual de individuos que se criaron en la Galicia franquista y de individuos que crecieron en la Galicia democrática, por lo que los resultados sobre el efecto de la edad deben interpretarse con cautela. Sería necesario ampliar la muestra para llegar a conclusiones más robustas sobre la vigencia de la asociación de patrones prosódico-pragmáticos más propios del castellano con el habla más formal entre los jóvenes gallegos. 


\section{Conclusión y direcciones futuras}

El objetivo de esta investigación ha sido determinar las configuraciones tonémicas de las interrogativas absolutas en el castellano de Galicia y examinar si su distribución se rige por el contexto pragmático - tal como ocurre en el castellano de Madrid - o si se observa variación por factores sociales relacionados con el contacto histórico entre castellano y gallego en esta región de España. Los resultados revelan que entre aquellos hablantes cuyos padres y abuelos provienen de otras regiones de España o de zonas urbanas de Galicia, las interrogativas absolutas presentan configuraciones tonémicas similares - y con una distribución pragmática también semejante - a las encontradas en Madrid. En cambio, entre aquellos hablantes con un origen familiar más arraigado en la región se encuentran configuraciones tonémicas similares a las de Madrid, pero con un uso pragmático diferente. En particular se observa que el tonema descendente (propio de preguntas confirmatorias o imperativas en Madrid) y el tonema circunflejo (propio de vocativos y peticiones insistentes en Madrid) se usan en el contexto neutro de preguntas informativas en el castellano de Galicia. La explicación que este trabajo ofrece para esta diferencia en el uso de los tonemas descendente y circunflejo es el contacto histórico con el gallego. La extensión del uso pragmático de los tonemas descendente y circunflejo en el castellano de Galicia es consistente con el modelo de cambio por contacto interlingüístico propuesto por Silva Corvalán (1994), ya que se trata de un cambio que no altera radicalmente la estructura de la lengua, sino que resulta en la eliminación de un contraste prosódico-pragmático.

La eliminación de este contraste que, recordemos, no es categórica entre los castellanohablantes de la presente muestra, sino que se limita a aquellos con mayor exposición al gallego, ha dado lugar a variación de tipo estilístico por la que el grado en que ocurren relaciones prosódico-pragmáticas más o menos agallegadas varía según la formalidad de la tarea. De los 19 hablantes que variaron entre H\%, L\% y HL\% en las preguntas informativas en el habla semi-espontánea, 10 usaron solamente $\mathrm{H} \%$ para el mismo contexto pragmático en una tarea más formal. Este resultado apunta a la pervivencia en Galicia de la estigmatización sociolingüística de los rasgos más agallegados del habla.

La presente investigación se limita a investigar uno de los aspectos en que el gallego ha influido en el castellano hablado en Galicia: la distribución de las configuraciones tonémicas de las interrogativas absolutas. Queda el interrogante de si aquellos hablantes con mayor influjo del gallego emplean otras estrategias para comunicar el contraste pragmático que se pierde con la extensión del uso del tonema descendente a las preguntas informativas. De igual modo cabe preguntarse si la variación estilística y por origen geográfico observada en este estudio también ocurre en otras modalidades oracionales. 
La respuesta a estas preguntas requiere de mayor elaboración y se aplaza para investigaciones futuras.

Además de añadir evidencia a la hasta ahora escasa literatura sobre el cambio de relaciones prosódico-pragmáticas por contacto interlingüístico, la presente investigación es la única hasta el momento que, mediante una metodología sociofonética, ofrece un análisis cuantitativo de los efectos a nivel entonacional del contacto histórico entre gallego y castellano. Existe la intuición entre hablantes del español europeo que la entonación distingue a los hablantes de Galicia de los de otras variedades peninsulares. El estudio experimental de las particularidades de dicha entonación es aún limitado, pero confiamos en que este trabajo contribuya a estimular futuras investigaciones en este campo.

Apéndice A

Ejemplos de combinaciones léxicas y los contextos en los que se presentaron:

(1)Estás hablando con tu hermana y le quieres decir que has hablado con vuestra madre. Le dices: llamó mamá.

(2)Estás hablando con tu hermana sobre los familiares que van a venir en avión a tu boda. Le dices: volaba Miguel.

(3) Entras a casa y le preguntas a tu madre si llegó tu hermana ya. Le preguntas: ¿Llegó Lola?

(4) Tu amiga te cuenta sobre la boda a la que tú no pudiste ir. En la boda hubo algunas personas dieron discursos y felicitaron a los novios. Quieres saber quiénes hablaron. Le preguntas: ¿Habló Manola?

Apéndice $B$

Contextos de interrogativas absolutas en la encuesta de situaciones, adaptada para Galicia a partir del modelo de Prieto y Roseano (2010).

Preguntas informativas

18 Entras en una tienda y le preguntas al dependiente si tiene mermelada.

19 Estás en la calle y pides hora.

20 Pide permiso para entrar en la sala donde te espera el médico.

21 Llamas por teléfono a casa de una amiga que se llama María pero no está. Más tarde llamas de nuevo pero ella no coge el teléfono, ¿cómo preguntas si ya llegó? 
22 Organizas una comida y has decidido cambiar la fecha para que todos los invitados puedan ir. Pregunta si podrán venir si la comida es el primer domingo de mayo.

Preguntas confirmatorias

30 Juan dijo que iba a venir a merendar pero quieres confirmarlo. ¿Qué le dices a Juan? (busca confirmación).

31 Antes de ir a trabajar tu hermano dijo que no se sentía muy bien. Al volver, lo encuentras en la cama temblando de frío. Ves que no se encuentra bien, pero se lo preguntas sabiendo cuál va a ser la respuesta. (Cercano a la negación, eh o verdad en posición final).

32 Tienes mucha ilusión que alguien venga a una cena que organizas. Se lo pides de manera que no pueda decir que no. (Cercano a la afirmación, eh o verdad en posición inicial).

33 Sabes que afuera hace mucho frío. Entra alguien bien abrigado y le preguntas si tiene frío. (Carácter hipotético, margen para responder sí o no).

Preguntas imperativas

34 Tus nietos hacen mucho ruido y no te dejan oír las noticias (en la televisión/radio). Les pides que se callen (ruego-orden).

35 No te hacen caso y esta vez lo pides más enfadada (orden).

36 Le pides a un amigo si quiere venir a tomar una limonada contigo (invitación)

37 Pide a tus sobrinos si quieren caramelos (invitación).

38 Organizas una fiesta en tu casa y tienes muchas ganas que un compañero tuyo vaya. Pídele si quiere venir (intención exhortativa: “me gustaría mucho que vinieses...”).

39 Necesitas subir tres pisos porque te dejaste el bolso (cartera si el informante es hombre) arriba. Vas con un niño pequeño y para ganar tiempo lo dejas abajo. Dile que no se mueva. (ruego-orden, con partícula eh).

40 Necesitas tranquilidad pero estás en medio de un gran alboroto. Pregunta si alguna vez habrá tranquilidad en este hogar.

Recibido: $24-V-2011$

Aceptado: 15-XI-2011 


\section{Referencias bibliográficas}

Aguilar, L., C. De la Mota y P. Prieto (coords.) (2009): Sp_ToBI Training Materials: http://prosodia.upf.edu/sp_tobi/ (15-2-2011).

Beswick, J. (2007): Regional nationalism in Spain: Language use and ethnic identity in Galicia. Bristol, Multilingual Matters.

Beswick, J. (2010): "Linguistic ideology in Galician factual radio broadcasts: Towards the castilianisation of Galician pronunciation traits?" En Lorenzo-Dus, N. (ed.): Spanish at work: Analysing institutional discourse across the Spanish-speaking world. London, Palgrave Macmillan, págs. 35-49.

Bouzada Fernández, X. M. (2003): "Change of values and future of the Galician language”, Estudios de Sociolingüística, 4(1), págs. 321-341.

Castro, O. (2003): "Pitch accent in Galician Spanish." En Sayahi, L. (ed.): Selected Proceedings of the First Workshop on Spanish Sociolinguistics. Somerville, MA, Cascadilla Proceedings Project, págs. 43-52.

Cestero, A. M. ${ }^{a}$, I. Molina y F. Paredes (2008): "Sociolinguistic issues of Madrid", International Journal of the Sociology of Language, 193/194, págs. 91-108.

Escandell Vidal, V. (1999): "Los enunciados interrogativos: Aspectos semánticos y pragmáticos.” En Bosque, I. y V. Demonte (eds.): Gramática descriptiva de la lengua española, Vol. 3. Madrid, Espasa, págs. 3929-3991.

Estebas, E. y P. Prieto (2009): "La notación prosódica del español: una revisión del Sp_ToBI”, Estudios de Fonética Experimental, XVII, págs. 263-283.

Estebas, E. y P. Prieto (2010): “Castilian Spanish intonation”. En Prieto, P. y P. Roseano (eds.): Transcription of Intonation of the Spanish Language. Munich, Lincom, págs. $17-48$.

Face, T. (2008): The intonation of Castilian Spanish declaratives and absolute interrogatives. Munich, Lincom.

Fernández Rei, E. (2005): "Situacion actual das investigacions prosodicas sobre o galego”, Bollettino dell'Atlante Linguistico Italiano, 29, págs. 147-159.

Fernández Rei, E. (2007): "On the transcription of Galician intonation". Ponencia presentada en el Third Phonetics and Phonology in Iberia (PaPi) Conference. Braga, Portugal, Junio de 2007.

Fernández Rei, E., A. Escourido Pernas, M. Caamaño Varela y L. Xuncal Pereira (2005): "A entoación dunha fala de Santiago: fronteira prosódica entre suxeito e predicado", Estudios de Fonética Experimental, XIV, págs. 141-165.

García, C. (1976): "Interferencias lingüísticas entre gallego y castellano", Revista Española de Lingüística, 6, págs. 329-343.

García, C. y L. Blanco. (1998): El castellano de Galicia: interferencias lingüísticas entre 
gallego y castellano. Madrid, Anaya.

Henriksen, N. (2010): Question intonation in Manchego Peninsular Spanish (Tesis doctoral). Universidad de Indiana, Bloomington, IN.

Labov, W. (1972): Sociolinguistic Patterns. Filadelfia, University of Pennsylvania Press.

Labov, W. (2001): "The anatomy of style-shifting". En Eckert, P. y J. R. Rickford (eds.): Style and sociolinguistic variation. Cambridge, Cambridge University Press, págs. 85-108.

López Bobo, Ma J. y M. Cuevas Alonso. (2009): “¿Prosodia norteña o castellana? Aproximación a la entonación del oriente de Cantabria”, Estudios de Fonética Experimental, XVIII, págs. 215-236.

López Bobo, Ma J. y M. Cuevas Alonso (2010): “Cantabrian Spanish intonation”. En Prieto, P. y P. Roseano (eds.): Transcription of Intonation of the Spanish Language. Munich, Lincom, págs. 49-85.

López Bobo, Mª J., M. Cuevas Alonso, L. Díaz Gómez y Mª D. Viejo Luciovillegas. (2008): "Análisis contrastivo de la prosodia del asturiano central y occidental", Language Design, 2, págs. 267-282.

Monteagudo, H. y A. Lorenzo (dirs.) (2006): A sociedade galega e o idioma. A evolución sociolingüística de Galicia (1992-2003). Santiago de Compostela, Consello da Cultura Galega.

O'Rourke, B. (2006): "Language contact between Galician and Spanish: Conflict or harmony? Young people's linguistic attitudes in contemporary Galicia". En MarMolinero, C. y M. Stewart (eds.): Globalization and Language in the SpanishSpeaking World: Macro and Micro Perspectives. Londres, Palgrave Macmillan, págs. 178-198.

Pierrehumbert, J. B. (1980): The phonetics and phonology of English intonation. Tesis doctoral, MIT.

Porto Dapena, A. (2001): "El español en contacto con el gallego". Ponencia en el II Congreso Internacional de la Lengua Española, Valladolid, Octubre de 2011: http://congresosdelalengua.es/valladolid/ponencias/ (7-10-2010)

Potowski, K. (2008): “'I was raised talking like my mom': The influence of mothers in the development of MexiRicans' phonological and lexical features". En Rothman, J. y M. Niño-Murcia (eds.): Linguistic identity and bilingualism in different hispanic contexts. Ámsterdam, John Benjamins, pñags. 201-220.

Prieto, P. y P. Roseano (coords.) (2010): Atlas interactivo de la entonación del español. http://prosodia.upf.edu/atlasentonacion/ (4-11-2010).

Ramallo, F. (2007): "Sociolinguistics of Spanish in Galicia", International Journal of the Sociology of Language, 184, págs. 21-36.

Regueira Fernández, X. L. (2004): “Estándar oral”. En Álvarez, R. y H. Monteagudo 
(eds.): Norma lingüística e variación. Unha perspectiva desde o idioma galego. Santiago de Compostela, Consello da Cultura Galega, Instituto da Lingua Galega, págs. 69-96.

Silva Corbalán, C. (1994): Language contact and change: Spanish in Los Angeles. Oxford, Oxford University Press. 